\title{
On the State and Stability of Fuel Cell Catalyst Inks
}

Shalmali Bapat ${ }^{1}$, Christopher Giehl ${ }^{2}$, Sebastian Kohsakowski ${ }^{3}$, Volker Peinecke ${ }^{3}$, Michael Schäffler ${ }^{2}$, Doris Segets ${ }^{1,4^{*}}$

${ }^{1}$ Process Technology for Electrochemical Functional Materials, Institute for Combustion and Gas Dynamics - Reactive Fluids (IVG-RF), University of Duisburg-Essen (UDE), Duisburg, Germany

${ }^{2}$ Anton Paar Germany GmbH, Ostfildern, Germany

${ }^{3}$ ZBT, The Hydrogen and Fuel Cell Center, Duisburg, Germany

${ }^{4}$ Center for Nanointegration Duisburg-Essen (CENIDE), Duisburg, Germany

\section{*Corresponding author:}

Doris Segets

E-Mail address: doris.segets@uni-due.de

Phone: +492033798230

Fax: +49 2033794195

Postal address: Institute for Combustion and Gas Dynamics - Reactive Fluids (IVG)

University Duisburg-Essen (UDE), NanoEnergieTechnikZentrum (NETZ), Carl-Benz-Straße 199, 47057 Duisburg, Germany 


\begin{abstract}
Catalyst layers (CL), as an active component of the catalyst coated membrane (CCM), form the heart of the proton electrolyte membrane fuel cell (PEMFC). For optimum performance of the fuel cell, obtaining suitable structural and functional characteristics for the CL is crucial. Direct tuning of the microstructure and morphology of the CL is non-trivial; hence catalyst inks as CL precursors need to be modulated, which are then applied onto a membrane to form the CCM. Obtaining favorable dispersion characteristics forms an important prerequisite in engineering catalyst inks for large scale manufacturing. In order to facilitate a knowledge-based approach for developing fuel cell inks, this work introduces new tools and methods to study both the dispersion state and stability characteristics, simultaneously. Catalyst inks were prepared using different processing methods, which include stirring and ultrasonication. The proposed tools are used to characterize and elucidate the effects of the processing method. Structural characterization of the dispersed particles and their assemblages was carried out by means of transmission electron microscopy. Analytical centrifugation (AC) was used to study the state and stability of the inks. Herein, we introduce new concepts, $S$ score, and stability trajectory, for a time-resolved assessment of inks in their native state using AC. The findings were validated and rationalized using transmittograms as a direct visualization technique. The flowability of inks was investigated by rheological measurements. It was found that probe sonication only up to an optimum amplitude leads to a highly stable colloidal ink.
\end{abstract}

\title{
Keywords
}

Analytical centrifugation, PEMFC catalyst ink, Dispersion, Stability trajectory, Rheology 


\section{Introduction}

As a sustainable and practical alternative to the internal combustion engine, few options tick all boxes for necessary characteristics. Among those in contention, proton exchange membrane fuel cell (PEMFC), which directly converts hydrogen fuel into electrical energy, remains a frontrunner. In its illustrative history of development, PEMFCs have made significant strides in achieving high energy efficiency, high power density, low operating temperature, and superior durability $[1,2]$. Despite the perceived progress, to meet the technical targets for widespread commercialization, there is a need for improvements on several fronts including — performance, durability, as well as on automated manufacturability for large scale production. To cover the remaining milestones in enhancing the performance and improving the durability, every component and critical process along the fuel cell design chain must be exploited.

The membrane electrode assembly (MEA), which makes up the heart of a PEMFC, is subject to numerous improvements from structural, chemical and performance perspectives. The MEA consists of (i) anode and cathode catalyst layers (CLs), (ii) polymer electrolyte membrane, and (iii) gas diffusion layers (GDLs). CLs are the home to the critical oxygen reduction reaction (ORR) and hydrogen oxidation reaction (HOR). In particular, the cathode catalyst layer (CCL), which facilitates the ORR is a 'bottleneck', due to limitations with the sluggish reaction rate. This makes the CCL the performance and cost controlling component of the PEMFC. As a solution, increasing platinum $(\mathrm{Pt})$ catalyst loading can accelerate the reaction, but limitations in scalability and economic feasibility restrict engineering of high Pt loading. As a result, numerous studies on PEMFCs have focused on the development of optimized catalyst coated membranes (CCM) and MEA layout with high catalyst utilization applying strategies such as (i) increasing Pt loading [3- 
5], and (ii) graded catalyst loading [6,7]. Yet, fulfilling the US Department of Energy (DOE) targets is an ongoing endeavor [8].

Toward this end, several recipes for catalyst ink dispersions have been tuned for optimum kinetic and thermodynamic performance [9-14]. The cathode of a high-power density PEMFC is typically made of a catalyst ink consisting of carbon black (C), forming an extensive skeleton. This high surface area framework is decorated by Pt particles as an electrocatalyst. A perfluorosulphonic acid, also commonly known as ionomer (e.g., Nafion $\left.{ }^{\circledR}\right)$ is additionally used to facilitate proton conduction and stabilize carbon aggregates / agglomerates. The fuel cell catalyst ink is a solid in liquid dispersion, but only ad interim. In operation, the catalyst ink is ultimately applied as a layer onto a substrate or a membrane. CLs are typically fabricated via solvent-based processing methods in which Pt/C catalyst powder is dispersed in a continuous phase containing solvent (mixture) and ionomer (see Figure 1B).

Traditionally, various solution-coating methods such as ultrasonic spray coating $[15,16]$, doctor blade [17-19], and large-scale roll-to-roll coating [20-22] have been employed to prepare CLs. More recently, inkjet printing [23,24], owing to its advantages over traditional techniques, has also been successfully experimented with, to fabricate MEAs. Supervising and controlling these MEA preparation methods to obtain the ideal microstructure and morphology of the CL is non-trivial, to the extent of being impractical for large scale production. This is especially challenging when there is very little known about the "best" CL structure. Hence, the penultimate stage - which is the ink dispersion becomes crucial, as there is a possibility of greater control on tuning the state and stability of the dispersion, in particular during scale-up. Together these have a direct consequence on desirable characteristics of the CL [25]. Therefore, knowledge-based development and 
"deployment" of catalyst inks can be facilitated by simultaneous investigation of dispersion state and stability.

The "dispersion state" describes (i) the chemical composition, (ii) the degree to which the solid phase is dispersed in the continuous phase, and (iii) characteristics of assemblages, such as their presence or absence, counts, or size distribution. Carbon materials themselves have a high propensity to form aggregates and agglomerates $[25,26]$. Since unequivocal compartmentalization of aggregate or agglomerate is challenging, without delving into the semantics, in this text, we refer to the presence of large supramolecular association of Pt decorated carbon with ionomer as "assemblages". The "dispersion stability" can be defined as the ability to resist aggregation, agglomeration, flocculation, coalescence, sedimentation, floatation or creaming. For complex, multi-material dispersions, several combinatorial interactions (i) within the dispersed phase(s) and (ii) between the dispersed and the continuous phase are pervasive. Certainly, dispersion state and stability are highly interconnected, and since both are dynamically interacting, these aspects change with time as well. Unraveling such multi-tier, complex interplays is crucial for controlling the dispersion behavior.

At its core, a poorly dispersed ink will result in aggregate and agglomerate formation, leading to disordered, ill-controlled layers during the fast-drying process. At the same time, the modified flow behavior of agglomerated dispersions often results in clogged spray or inkjet nozzles [27,28]. To avoid clogging of the nozzles and obtain ordered and continuous layers, the dispersions should provide sufficient colloidal stability. Moreover, the rheological behavior of catalyst ink is critical in the manufacturing of CLs. The flowability of ink governs the CL uniformity [22,29], thickness [30], and ink penetration into the substrate [31]. Thus, the colloidal state, stability, along with 
rheology, have an enormous influence on the coating characteristics, subsequent mass transport through GDLs, and ultimately the electrochemical performance.

Dispersion stability is a desirable trait to ensure a high-quality end-product. Reports show how certain ink characteristics can translate to maximization of the available Pt loading on the one hand and to positively influence the mass transport through the active layer on the other hand. There are two available 'knobs' to control dispersion state and stability, namely (i) ink composition and (ii) choice of processing methods. Several studies have reported the modifications to the ink recipe by changing the carbon-support type and ionomer to carbon (I/C) ratio. For instance, Soboleva et al. systematically investigated the effects of ionomer loading on the water sorption and electrochemical activities of two types of carbon [32]. Khandavalli et al. provided insights on the rheological behavior of ink varying I/C ratio and carbon-support type [33]. Similarly, different solvent selection has been explored, which results in maximum stability [22,28], and electrochemical performance $[14,34]$. It must be noted that a good solvent for carbon only may not be good enough for the mixture of Pt/C with an ionomer. Previous studies report the significant impact of the state of the ionomer on the MEA properties [25,35-37]. The complex interplay of ionomer and carbon at particle and molecular levels using Derjaguin-Landau-Verwey-Overbeek (DLVO) theory [22,28], molecular dynamic simulations [10,38], and adsorption isotherms [39] has been explored in several reports. Furthermore, the seminal work by Pollet and Goh highlights the effects of processing methods such as ultrasound and shear mixing on the electrochemical activity and the particle size of catalyst inks [40,41].

Many technologies together provide a set of tools that enable useful characterization of structural and chemical components of the ink, to systematically study the effects of changes in recipe or processing. In order to describe the state, electron microscopy is widely used. Herein the images 
provide a localized view of the pore, void, and aggregate formation. But imaging relies on physical modifications to the ink, such as drying [42]. Not to forget, the subjectivity in image detail selection and field of view. Dynamic light scattering (DLS) allows the sizing of particles in the dispersion. But it suffers from low resolution for broadly distributed systems and very often requires a high degree of dilution, thus distorting the particle sizing analysis [43]. Small-angle or wide-angle $x$-ray scattering analysis is a powerful technique for characterizing inks $[16,33,44]$; however, it is associated with challenging data interpretation [42] and higher operating and investment costs. Despite the options available in-store, a gap in tools and technologies to study ink dispersions in their native state and at operational concentration levels exists. Moreover, concurrent investigation of catalyst ink dispersion state and stability remains an opportunity to be exploited.

Analytical centrifugation (AC) is a non-destructive technique to study dispersions, in which small sample volumes are exposed to higher g-force, to study particle migration behavior. Over the years, AC owing to its quick and easy preparation demands and economic advantages has widely been used in many research fields such as food, pharmaceutical, and coating industries. For instance, Mert demonstrated the potential of AC to evaluate the physical properties of ketchup products [45]. Optimized formulations of solid lipid nanoparticles (SLNs) for essential oils were developed by Zielińska et al. using AC [46]. Barrios reported the applicability of AC to estimate the shelflives of polymeric coatings [47]. For fuel cell applications, Hoffman et al. [19] made first studies based on simplified ink formulations using AC. They employed AC to study different I/C ratios, but final ink dispersions were prepared sans Pt. We have also begun to address the challenges in investigating inks and fill the gaps using AC by proposing a novel technique to evaluate the dispersion state using spatio-temporal maps, called transmittograms [48]. However, systematic 
evaluation of process-structure relations through a time-resolved assessment of ink behavior and stability has not been explored to the best of our knowledge.

In this work, we demonstrate several possibilities for qualitative and quantitative assessment of the catalyst inks, by employing a table-top AC device, namely LUMiSizer®. We propose new methodologies for data mining and evaluation of AC measurement data. Using a novel approach of transmittogram analysis to study sedimentation dynamics, we show how to elicit information on the inherent dispersion characteristics and track the assemblages. Along with the state, for simultaneous assessment of stability, we introduce a new concept called "stability trajectory". A stability trajectory is obtained by calculating an "S score" for each time point of the AC run. We choose four different methods to prepare ink dispersions and compare procedural differences using transmittograms and stability trajectories. Most importantly, all the investigations are made at technical ink concentrations, without altering their native state. The experimental workflow is structured into four parts. First, a catalyst powder is dispersed into a solvent mixture by four processing methods. Secondly, through transmission electron microscopy (TEM) imaging, structural characteristics of the ink are observed. Thirdly, inks are measured using AC. And lastly, rheological characterization of the inks is performed to assess handling properties and solutioncoating performance.

\section{Theory and Praxis}

In this section, we show how AC measurement data are recorded. We describe the newly proposed techniques of transmittograms and stability trajectories, show how to evaluate and interpret them. 


\subsection{Principle of analytical centrifuge}

The analytical photocentrifuge LUMiSizer® (LUM GmbH, Berlin, Germany), is a cuvette-based centrifuge working on the principle of STEP-technology ${ }^{\mathrm{TM}}$ [49]. As the rotor moves, the sample sedimentation is accelerated because of centrifugal force. The rectangular sample cell is fixed horizontally onto a rotor plate. Monochromatic light is pulsed through the sample cell, and the attenuated optical transmission through the colloidal dispersion is captured by an array of chargecoupled device (CCD) detectors (see Figure 1A). Under the effect of centrifugal force, particles migrate across the length of the sample cell, i.e., from $r_{i}$ to $r_{f}$. The distance moved by the sample is expressed as radial distances, i.e., distance from the rotor center. The bottom of the sample cell is around $129 \mathrm{~mm}$.

The transmitted light is captured at a fixed interval of time, where the total time of experiment and time interval for the measurement are both user-defined parameters. Higher levels of attenuation correspond to the larger local volume fraction of the dispersed phase. Conversely, low levels of attenuation correspond to a smaller local volume fraction. Hence, the resulting transmission profiles or fingerprints as a function of space and time allow us to track the migration of particles. The measurement data captured by the instrument software is in the form of a matrix with rows corresponding to values at each space point and columns corresponding to each time point. These raw data serve as the basis for both instrument software evaluation features and the methods proposed in this work. Among the different out of the box features of the software, herein we primarily use two modes - (i) qualitative assessments using fingerprints, and (ii) quantitative assessments using instability index $[46,50,51]$. The procedures performed by the manufacturer's software for computing instability index [52], are described in the supporting methods. Owing to 
the limitations of these modes in usability and interpretability, we turn to novel data processing methods.

\subsection{Transmittogram analysis}

In our previous work, as shown in Figure 1C, we demonstrated how the raw data acquired by $\mathrm{AC}$ can be transformed into intuitive visual formats called transmittograms [48]. Such an image is constructed by plotting a heatmap from all captured instantaneous transmission values over space across the sample cell and time. The $x$ - axis of the plot shows the time of the experiment. The $y$ - axis shows the radial position of the sample content, which typically spans from radial positions $\sim 103$ to $\sim 130 \mathrm{~mm}$. A heatmap is constructed using grey values between 0 and 255 assigned to the transmission. The change in the shades of the color indicates changes in the transmission and / or particle concentration. The darkest color signifies the lowest transmission values, indicating the highest concentration, and the brightest color relates to the highest transmission value implying the lowest concentration. Assignment of color values can be done in various ways to achieve the best visual contrast. Distinguishing features arising from transmission gradients can be facilitated using color mapping to defined intervals calculated based on the quantiles of the data.

The ability to visualize the entire centrifugation experiment while tracking several colloidal features can be a powerful tool in the study of complex dispersions. Different types of qualitative and quantitative information can be drawn from such visual maps. Sample related features such as the meniscus and sedimentation fronts can be identified by boundaries seen in the image due to sharp transmission gradients. For example, three shades of grey color in the schematic plot in Figure 1C indicate the presence of particles of three different sizes settling at different times. With the transmittogram, the user can instantly fetch information regarding the transient sedimentation state of assemblages and the progression of the sedimentation front. The exact time at which 
complete settling has been achieved can be extracted easily. Moreover, particle sizes can also be estimated using Stokes' law. Collectively, transmittograms lend heavily to deducing the dispersion state and open avenues for deriving the colloidal stability characteristics.

Noticeably, a quantitative appraisal for the dispersion stability cannot be made from a visual analysis of images. During heatmap plotting, several dispersion features can be confounded or missed by the human eye due to inadequate color mapping. In pursuit of a quantitative evaluation of the destabilized state of the dispersion, we turn to calculation of stability metrics. This facilitates objective comparison between samples based on their colloidal stability. 


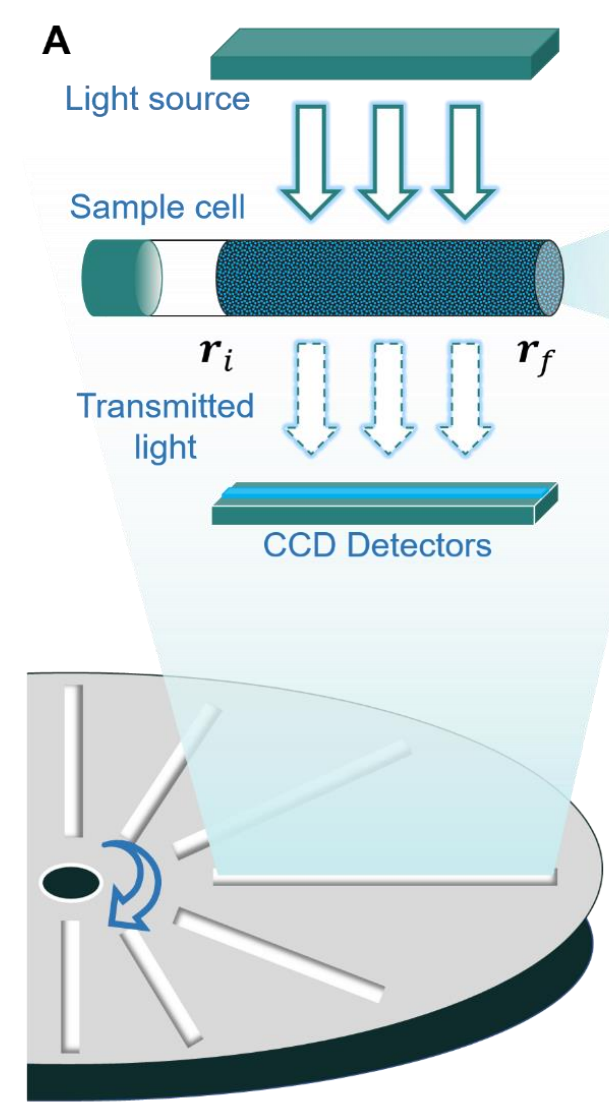

Analytical centrifuge
B

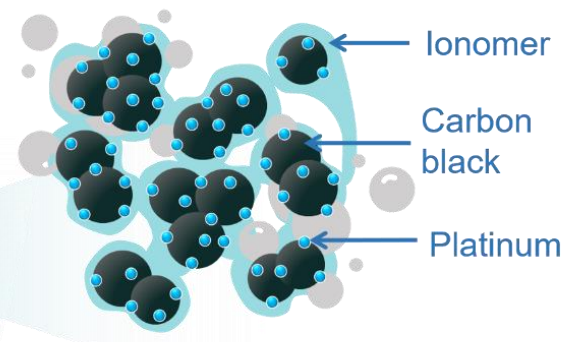

Ink structure
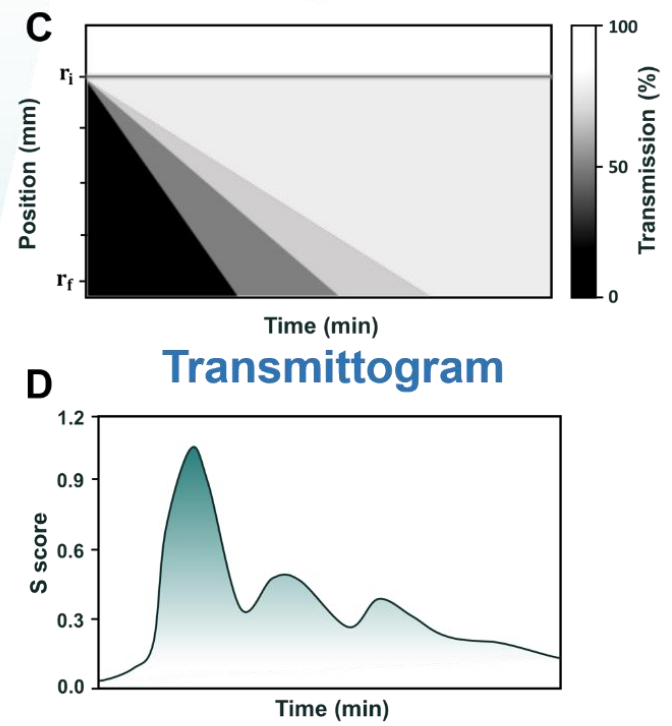

Stability trajectory

Figure 1: Schematic showing the novel characterization toolbox introduced in this work to study fuel cell inks using AC. (A) Working principle of AC. Light transmitted through the sample cell over a radial position of $r_{i}$ to $r_{f}$ from the rotor center is captured by CCD detectors at defined time intervals. (B) Schematic structure of a PEMFC ink composed of a carbon-supported Pt catalyst and ionomer. (C) Transmittogram illustrating the time and space resolved changes in attenuated transmission values. The initial $r_{i}$ and the final $r_{f}$ radial position of the sample from the rotor center and elapsed time are indicated on the $y$ - and $x$-axes, respectively. A grey value is assigned to instantaneous transmission values to construct the heatmap. (D) Stability trajectory illustrating the S score derived from instantaneous transmission values over elapsed time. 


\subsection{S score}

The new metric called $\mathrm{S}$ score is a measure of stability as a function of time, based on the differences in transmitted light. Here stability of the dispersion is derived from its sedimentation or clarification patterns, captured in the form of light transmitted across the length of a sample cell at fixed intervals of time. The transmission profile $\left(T_{i}\right)$ is first normalized by subtracting the corresponding mean $\bar{T}_{l}$, across the radial positions, and dividing by the standard deviation $\left(\sigma_{T_{i}}\right)$ of the profile. This is also known as z-normalization (Equation 1). The resultant normalized transmission profile $\left(Z_{i}\right)$ is used for further calculations:

$$
Z_{i}=\frac{T_{i}-\bar{T}_{l}}{\sigma_{T_{i}}}
$$

Z-transformation ensures all data are scaled uniformly. Further, the median of each normalized profile is calculated (Equation 2):

$$
\begin{aligned}
& \widetilde{Z_{l}}=\operatorname{median}\left(Z_{i}\right) \\
& \widetilde{S_{l}}=\operatorname{median}\left(\left|Z_{i}-\widetilde{Z_{l}}\right|\right)
\end{aligned}
$$

Following this, the median absolute deviation (MAD) is calculated for each of the transmission profiles, resulting in an S score $\left(\widetilde{S_{l}}\right)$ for each time point (Equation 3). The S score concept involves the calculation of MAD estimator over time. Statistically, MAD is a robust measure of variance. S score values are always positive, as mathematically, they are calculated based on the absolute values.

$\mathrm{S}$ score of a sample at a particular time shows the extent of variation in transmission values across the sample cell. A lower S score indicates a stable state of the sample, because the dispersed phase is evenly distributed along the cell. Conversely, a higher S score means an unstable state of the 
sample due to heterogeneity upon sedimentation. Here we briefly revisit the concept of heterogeneity. The reader is cautioned that in this paper, when heterogeneity is discussed, it is not implying the polydispersity or multi-material nature of the sample. Instead, it refers to the heterogeneous distribution of the relative motion of particles, i.e., some particles are separating faster than others. As a result, the measured transmission values across the sample cell also show large variations and distinct gradients, which are captured by the $\mathrm{S}$ scores.

Notions of dispersion stability can be multifaceted, i.e., related to electrostatic interactions or settling behavior, among others. The $\mathrm{S}$ score value itself is a surrogate metric for tracking stability based on the quantitative assessment of settling. Further, when the $\mathrm{S}$ scores are tracked over a period of time, resulting in a trajectory (see next section), it can be indicative of the evolution of the heterogeneity of the dispersed phase.

The S scores developed in this work and the instability index provided by the SepView@ software package (see supporting methods) are fundamentally different in four ways. First, the value of the instability index at any time is the characteristic measure of the instability of the sample. This is because the instability index is calculated as a difference to the transmission of the first profile, i.e., it is in comparison to the initial state of the dispersion. In contrast, the $\mathrm{S}$ score at a particular time point represents the instantaneous state of the sample, derived from the dissimilitude of the transmission values across the sample cell at a specific time point, independent of the system's initial state. Secondly, the instability index depends on the selection of a region of interest (ROI). S score calculation does not require a preselection of ROI, albeit an ROI can be used. Thirdly, the instability index is normalized against the maximum clarification achieved by the sample. This depends on the attenuated transmission levels of the last profile. By default, a value of $90 \%$ transmission is often used as the mean transmission for the last profile. The $\mathrm{S}$ score evaluation 
does not rely on normalization against maximum clarification. Fourthly, for optimum comparison of sample stabilities using instability index, all samples must be run simultaneously. Even if the samples cannot be run together, care must be taken to ensure the same operating procedure during the measurement process. By contrast, the data normalization step enables $\mathrm{S}$ scores to compare any (reasonable) samples, even in cases where one sample is run for a shorter experimental time than another sample (but with the same relative centrifugal force (RCF)). Altogether, and as will be discussed in the following sections, $\mathrm{S}$ scores can provide valuable and deep insights on the dispersion state and stability of a sample and facilitate comparability between experimental data obtained from AC measurements, in general.

\subsection{Stability trajectory}

When S scores are plotted with time, the resulting trace is referred to as a stability trajectory (see Figure 1D). The shape of the trajectory is unique to the sedimentation behavior of the dispersion. The stability trajectory provides an indication of the extent of dispersion stability. An ascending trajectory indicates decreasing stability within the corresponding time period. This can be alluded to substantial extent of particle migration, aggregation, or agglomeration phenomena. Likewise, a descending trajectory indicates an increase in stability within the corresponding time period. As a whole, the stability trajectory is not a monotonically increasing trace. If there are several distinct peaks and valleys, then they are indicative of a diverse mix of particles, forming different sedimenting fronts of assemblages. A diverse mix of particles may not necessarily be due to the multi-material dispersed phase only, but also because of polydispersity or multimodality. Hence, a relatively noisy trajectory means that there are many diffused boundaries, either of the dispersed phase or continuous phase only. 
To explore the behavior of the trajectory after settling is completed and only the continuous phase is left, one can look at the stability trajectory for a solvent (see supporting Figure S1). Typically, such a trajectory is characterized with low values of $\mathrm{S}$ score. Besides, for samples that have no significant changes in transmission over time in a photocentrifuge, the stability trajectory will remain horizontal over time. This can happen in two scenarios - (i) when the dispersed phase has completely settled, and (ii) when a dispersion is highly stable and does not experience any sedimentation in the given experimental time. However, in the latter, the plateau will be characterized with higher S score values than the former, solvent-only samples.

The trajectory itself serves as a quantitatively rich tool for the comparison of sample stability. Alternatively, individual trajectory features such as ascending / descending rate, the time window for plateauing trace, times corresponding to peak or valley positions - can be directly used to evaluate and compare samples. The choice of relevant features can be rationalized based on the objective of the investigation. For example, readily discernible valleys can point towards complete settling of a specific assemblage (supporting Figure S2). Observations from the corresponding transmittograms help to establish and assign the meaning to distinct trajectory features. In the event when complete settling has not been achieved (e.g., supporting Figure S2B), in this work, we choose the corresponding time point for which the highest $\mathrm{S}$ score is obtained, as means of comparison. We refer to this time as the bulk sedimentation time (BST). Physically, this denotes that the distribution of transmission values beyond this time has significantly shifted towards higher values. In other words, more light is transmitted because a majority of the particles have undergone settling. Together, such features (e.g., time points corresponding to extrema) can be derived from automatic peak processing and fed into databases. 
While evaluation of features underscores the importance of the trajectory, not all features might be manifested or prove to be useful for sample comparison. Further, it must be noted that the parameters derived from peak processing of the trajectory can be compared for different samples when the RCF is the same for all the samples. Additionally, comparing peak parameters for samples with varying capture times can have higher uncertainty associated due to different "sampling" rates.

\section{Materials and Methods}

\subsection{Materials for preparing catalyst inks}

Commercial Pt/C catalyst (40 \% Pt on C) were purchased from Alfa Aesar. The Nafion® ionomer dispersion D521 (1100 equivalent weight, at 5 wt. \%) was purchased from Ion Power. 2-propanol ( $\geq 99.0 \%$ purity, VWR international) and Millipore water $(18.2 \mathrm{M} \Omega \mathrm{cm}$ ) were used to prepare dispersions.

\subsection{Catalyst ink preparation and dispersion}

The cathode catalyst inks were prepared by mixing Pt/C powder, Nafion ${ }^{\circledR}$ dispersion, Millipore water, and 2-propanol (IPA) with an Ionomer/Carbon volume ratio of 0.9 (without Pt) and total $\mathrm{H}_{2} \mathrm{O} / \mathrm{IPA}$ volume ratio of $1.43 .20 \mathrm{ml}$ of the mixture were exposed to four dispersion procedures. The solid content was kept constant at $0.5 \mathrm{wt} . \%$ to prepare dispersions. The first ink was mixed using a magnetic stirrer (IKA 50/60 Hz, $50 \mathrm{~W}$ ) at $500 \mathrm{rpm}$ over 24 hours (ink sample MS). The second type of ink was mixed using an ultrasonic bath (Elmasonic S10, 50/60 Hz, $30 \mathrm{~W}$ ) for 30 min (ink sample UB). The third set of ink was prepared by 30 min of ultrasonic bath followed by 10 min of probe sonication (Bandelin HD2200) at an amplitude of $20 \%$ (2 min. ON and $1 \mathrm{~min}$. $\mathrm{OFF})(\mathrm{UB}+\mathrm{S} 20)$. The fourth ink was prepared by $30 \mathrm{~min}$ of ultrasonic bath followed by $10 \mathrm{~min}$ of 
probe sonication at an amplitude of $70 \%(2 \mathrm{~min}$. ON and $1 \mathrm{~min}$. OFF) (UB+S70). The used sonotrode tip was $5 \mathrm{~cm}$ long and had a diameter of $13 \mathrm{~mm}$. For all samples exposed to probe sonication, an ice bath was used to avoid the overheating of the sample.

\subsection{TEM imaging}

The structure of ink constituents subjected to the various dispersion techniques was characterized by a TEM (JEOL 2200FS). The diluted catalyst ink was drop cast on the copper grids and dried at room temperature. The images of the inks were acquired in bright field mode with an acceleration voltage of $200 \mathrm{kV}$.

\subsection{Measurements with the analytical centrifuge}

AC was performed using a LUMiSizer®, a multisample analytical centrifuge (LUM GmbH, Berlin, Germany). For measurements, approximately $0.4 \mathrm{ml}$ of sample was filled in a polycarbonate cuvette with an optical path length of $2 \mathrm{~mm}$. The temperature was set to $10{ }^{\circ} \mathrm{C}$, and a light source with a wavelength of $870 \mathrm{~nm}$ was used. A rotor speed of $4000 \mathrm{rpm}(2300 \times \mathrm{g}$ at the bottom $\sim 130 \mathrm{~mm}$ ) was applied. Every measurement consisted of 333 profiles, which were recorded every $175 \mathrm{~s}$. The total centrifugation time was $\sim 16$ hours. To check the reproducibility, three different ink samples were prepared on separate days for each dispersing method (i.e., 12 samples in total) and were measured in triplicate. Raw data (in the form of attenuated transmission values) from SepView®, were saved into a spreadsheet file format. The default setting of nine-point averaged transmission values was used.

\subsection{Evaluation of transmittogram and stability trajectory}

Scientific libraries in Python language were used for reading, processing, and evaluation of the AC data. Numerical processing was performed with NumPy [53] and Pandas [54], and plotting 
was done using Matplotlib [55]. Custom functions were written for reading and processing the raw data, automatic quantile-based color mapping, and generation of transmittograms. Additional functions were written based on Equations 1 to 3 to calculate the S scores. The stability trajectory was plotted using the time-series array of $S$ scores. The entire sample height was used in the calculation of S scores. In the current form, the code will be provided on request.

\subsection{Rheological characterization}

Rheological measurements were performed with an air-bearing Modular Compact Rheometer (MCR 302) from Anton Paar (Anton Paar GmbH, Graz, Austria) equipped with a water-cooled Peltier Temperature Device (P-PTD 200) and a cone-plate (CP) measuring geometry (CP60-0.5). The latter was selected according to the expected sample viscosities in the lower mPa.s range (based on pure solvent viscosity) and the dispersed solid fraction (0.5 wt. \%).

After sample loading and thermal equilibration, viscosity curves were measured at $25^{\circ} \mathrm{C}$ from shear rate $0.1 \mathrm{~s}^{-1}$ to $1000 \mathrm{~s}^{-1}$ with measuring point duration decreasing logarithmically from $50 \mathrm{~s}$ to $2 \mathrm{~s}$, with a resulting total test time of 285 s. A solvent trap was used to minimize sample evaporation. The repeatability and reproducibility were confirmed by duplicate measurements and testing three separately prepared ink samples for each dispersing method, i.e., 12 samples and 24 measurements in total.

\section{Results and Discussion}

\subsection{TEM imaging of inks}

TEM micrographs of ink dispersions after dilution of 1:1000 are shown in Figure 2. In the panel, the magnification increases from $60 \mathrm{Kx}$ (left panels), over $100 \mathrm{Kx}$ (middle panels), to $300 \mathrm{Kx}$ 
(right panels). TEM micrographs of inks made using the magnetic stirrer are not captured. These samples formed large agglomerates, causing blobbing onto the beaker walls as well. Images A to C belong to ink sample UB, followed by images D to F to ink sample UB+S20. Lastly, images $G$ to I belong to sample UB+S70. The images suggest that the applied sonication energy did not dislodge the Pt nanoparticles, which is a crucial aspect contributing to the ORR and thus controlling the electrode performance. Apart from showing the spread of Pt particles around the ionomer impregnated carbon, images reveal the presence of several fractal agglomerate structures. Areas in the micrograph with relatively low brightness represent the carbon agglomerates (encircled in white). Breaking the agglomerates and / or aggregates in a defined way is the desired outcome for any ink processing method.

Interestingly, it is hard to distinguish agglomerate breakup on using additional probe sonication treatment to the inks after ultrasonic bath treatment. What stands out is that the inks prepared using high amplitude probe sonication do not show a significant breakup of carbon support or dislodging of Pt nanoparticles. Surplus sonication often leads to the detachment of catalyst from carbon skeleton and ionomer network [16]. Although many reports describe assemblage characteristics using imaging and major progress is also observed in the area of TEM image analysis [56], only a limited distinction of voids, agglomerates, primary aggregates, among others, can be made from the images. Reasons are excessive dilution and potential drying artifacts resulting directly from the requirements for the TEM. Hence, images in Figure 2 display the aggregated state of inks, but only to some extent as the conditions during imaging are far from the actual state of inks as prepared at technical concentrations in dispersion. 

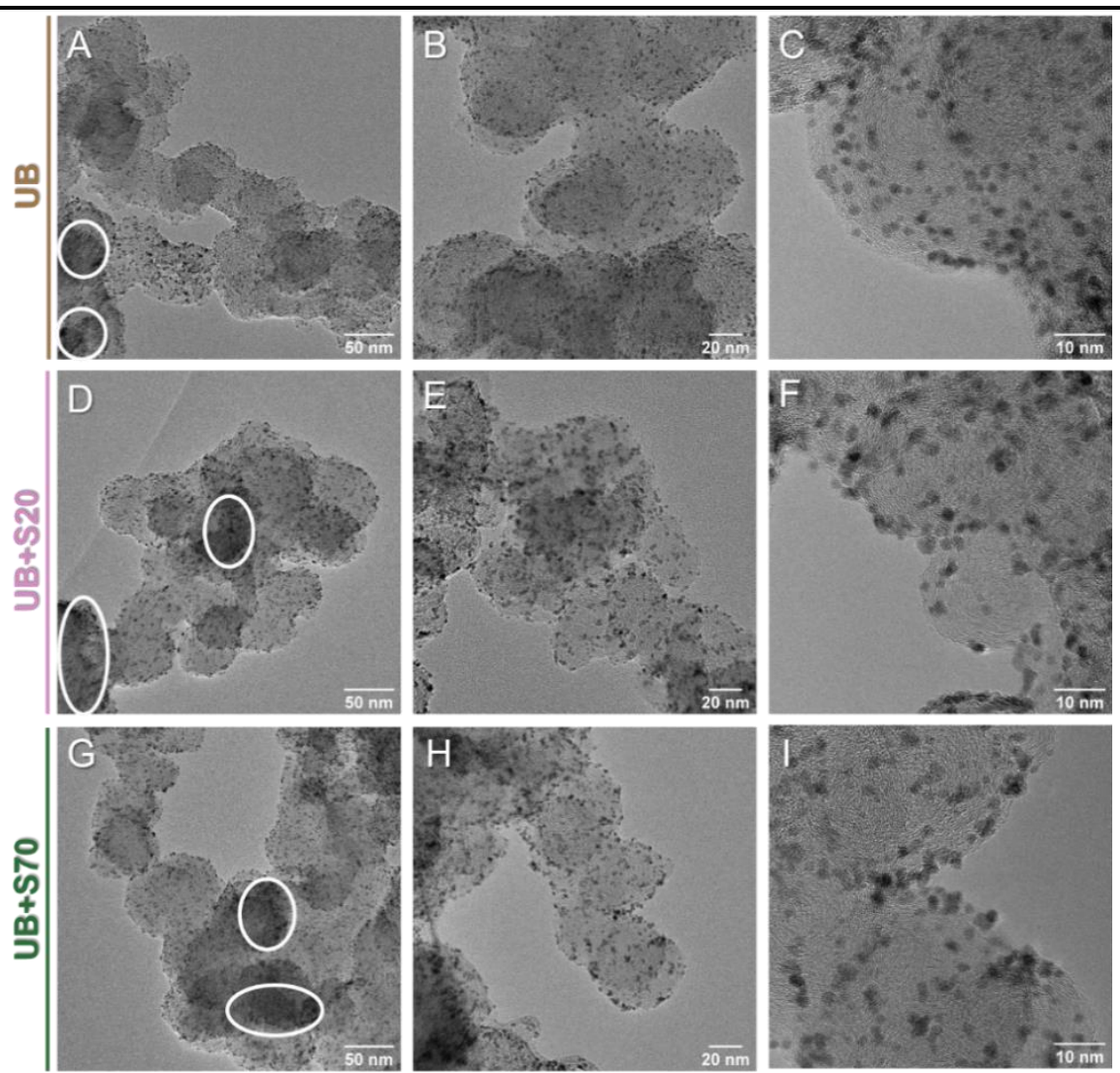

Figure 2: TEM micrographs at different magnifications, $60 \mathrm{Kx}$ (left panels), $100 \mathrm{Kx}$ (middle panels), and $300 \mathrm{Kx}$ (right panels). (A-C) Images of the catalyst ink sample UB. (D-F) Images of the catalyst ink sample $\mathrm{UB}+\mathrm{S} 20$. (G-I) TEM images of the catalyst ink sample $\mathrm{UB}+\mathrm{S} 70$. Some representative areas potentially covered with agglomerates are shown in white circles.

\subsection{Dispersion characteristics}

\subsubsection{Sedimentation fingerprints}

To address the main objective of this work, i.e., to fully characterize both the state and stability of catalyst ink, AC analysis was used. Transmission signals captured at a particular time, across the length of the sample cell, are referred to as transmission profiles or fingerprints. They constitute the main readout for the photocentrifuge. Representative fingerprints for inks MS, UB, UB+S20, $\mathrm{UB}+\mathrm{S} 70$ are shown in Figure 3. 

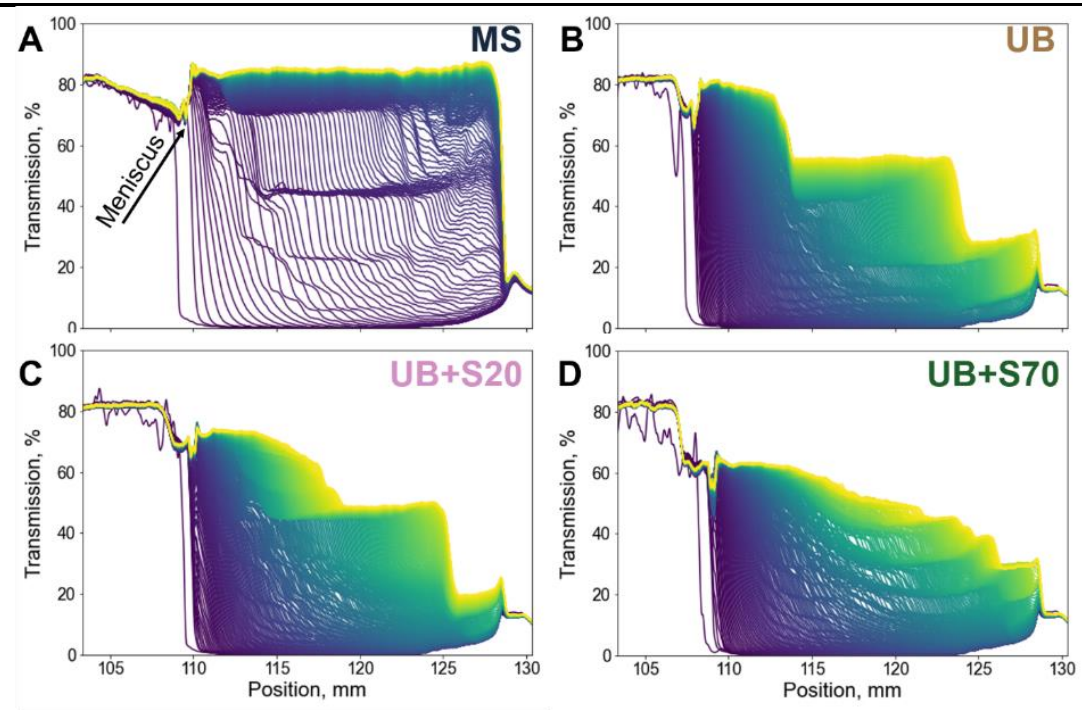

Figure 3: Transmission fingerprints for catalyst ink samples MS, UB, UB+S20, and UB+S70, respectively. The first profile is colored as violet, and the last profile as yellow. The meniscus for ink sample MS is shown by a black arrow at a radial position of $110 \mathrm{~mm}$. Inks were centrifuged at $4000 \mathrm{rpm}$ at $2300 \mathrm{~g}$ for about 16 hours.

Fingerprints provided by the software accompanying the instrument (SepView $®)$ show each line as a separate transmission profile. With the help of line color coding, the reader can get a general idea of the time progression of the experiment. Here the first profile is colored as violet and the last profile as yellow. Since all 333 profiles are plotted, except for ill-dispersed sample MS with fast sedimentation, the lines are very close to each other. In all cases, the distance between subsequent profiles is very narrow in the early onset of the sedimentation experiment and towards the end. This results in the lines forming compact segments of violet and indigo (start phase of the experiment), and of green and yellow (end phase of the experiment). The meniscus position is manifested by a small valley before the radial position of $110 \mathrm{~mm}$ from the rotor center. The transmission value before the meniscus indicates the transmission through the empty cuvette, which is $\sim 80 \%$ for all samples. What can be clearly identified is how the transmission increased 
in the middle portion of the measurement cells (from $115 \mathrm{~mm}$ to $120 \mathrm{~mm}$ ) for the samples MS, $\mathrm{UB}, \mathrm{UB}+\mathrm{S} 20$, and $\mathrm{UB}+\mathrm{S} 70$, respectively. We see that transmission for sample MS over the cuvette filling height is $\sim 85 \%$, indicating complete sedimentation has occurred.

Several published works report different motifs in the fingerprints and their corresponding interpretations on the particle interaction and migration behavior resulting from monodispersity, polydispersity, multimodality or solid concentrations [57,58]. Different particulate systems, in different solvents, varying concentrations, in the presence of additives, can have their behavior manifested in diverse ways. This is clearly seen for ink samples that no one motif is dominantly expressive of the underlying stability of the dispersion. What can be easily understood from the fingerprints is the highly convoluted nature of the transmission profiles, which form different but highly characteristic patterns that are hard to decode.

A typical motif in the form of steps is observed in the fingerprints (see Figure 3B-D). Few reports argue that the formation of such steps can allude to convective instabilities leading to the formation of layers during sedimentation. For instance, Butenko et al. [59] noticed the formation of successive plateaus, when they measured $\mathrm{Cu}-\mathrm{Ag}$ nanoparticles. It is reported that such convective instabilities arise primarily due to (i) temperature gradients across the cuvette and (ii) rectangular shaped sample cell. Uttinger et al. [60] recently reported the presence of temperature gradients of 0.1-50 $\mathrm{mK}$ across the sample cell, at higher instrument operation temperatures, which might lead to convective rolls for nanoparticle suspensions over long experimental times. In order to reduce the effect of lateral temperature gradients, all measurements were performed at low temperatures $\left(\sim 10^{\circ} \mathrm{C}\right)$. In the case of ink dispersions, our experience with their characteristics strongly shows that these motifs are not random occurrences. We consistently and reproducibly observe such plateauing profiles for samples with varied constituencies, concentrations, solvents, and measured 
at different time intervals and rotor speed and depending on the particular sample treatment history. Additionally, the propensity of carbon to form large agglomerates and flocs is well known $[25,26]$. These assemblages sediment at different rates, leading to the formation of several layers. Hence, it can be concluded that the formation of several layers during sedimentation is a direct outcome of agglomeration processes. It should be noted that stratification and layering can be either (i) preexisting from the very beginning of the measurement or (ii) are formed during the measurement. The former are true features of the sample, while the latter, although just evolving during the measurement, are also unique, characteristic, and strongly sample related.

In light of these observations, it is worthy to note that fingerprints for fuel cell inks do not provide holistic information on the ink dispersion. Collective conclusions possible until now are - (i) the location of the meniscus or air-liquid boundary can be seen in the fingerprints, (ii) for samples other than MS, complete sedimentation has not been achieved, and (iii) polydispersity motifs shed light on the presence of agglomerates. Thus, these data allow us to recognize expected dispersion characteristics, permitting us to advance to recently developed transmittograms analyses in the next section. This enables us to go beyond deducing from line patterns of fingerprints and directly "see" how the dispersion is.

\subsubsection{Transmittograms}

Figure 4A is the transmittogram for catalyst ink MS. The $x$-axis represents the time elapsed from the start of the experiment. In this case, the experiment was carried out for over 16 hours. The $y$ axis represents the height of the filling chamber of the sample cell. This length scale is reported as the radial distance from the rotor center. Hence the values start from $103 \mathrm{~mm}$ (top) to $129 \mathrm{~mm}$ (bottom). The meniscus or the air-liquid interface is seen around $109 \mathrm{~mm}$, through the course of the experiment. A sedimentation front is annotated in the figure. At least three such sedimentation 
fronts are observed, indicating the existence of at least three assemblages in the ink sample. Assemblage I settles in about 2 hours. The complete settling of particles takes place within 4 hours. When the sedimentation is complete, the liquid becomes clearer, as indicated by the lighter shade of grey, having transmission values above $70 \%$. Interestingly, the sedimentation front for assemblage III becomes diffused towards the lower end of the sample cell after 2 hours of experimental elapse time. This is because of the equalization of centrifugal force on the particle and other forces (diffusion forces plus buoyancy force) due to solvent interactions. This leads to a diffused sedimenting front. 

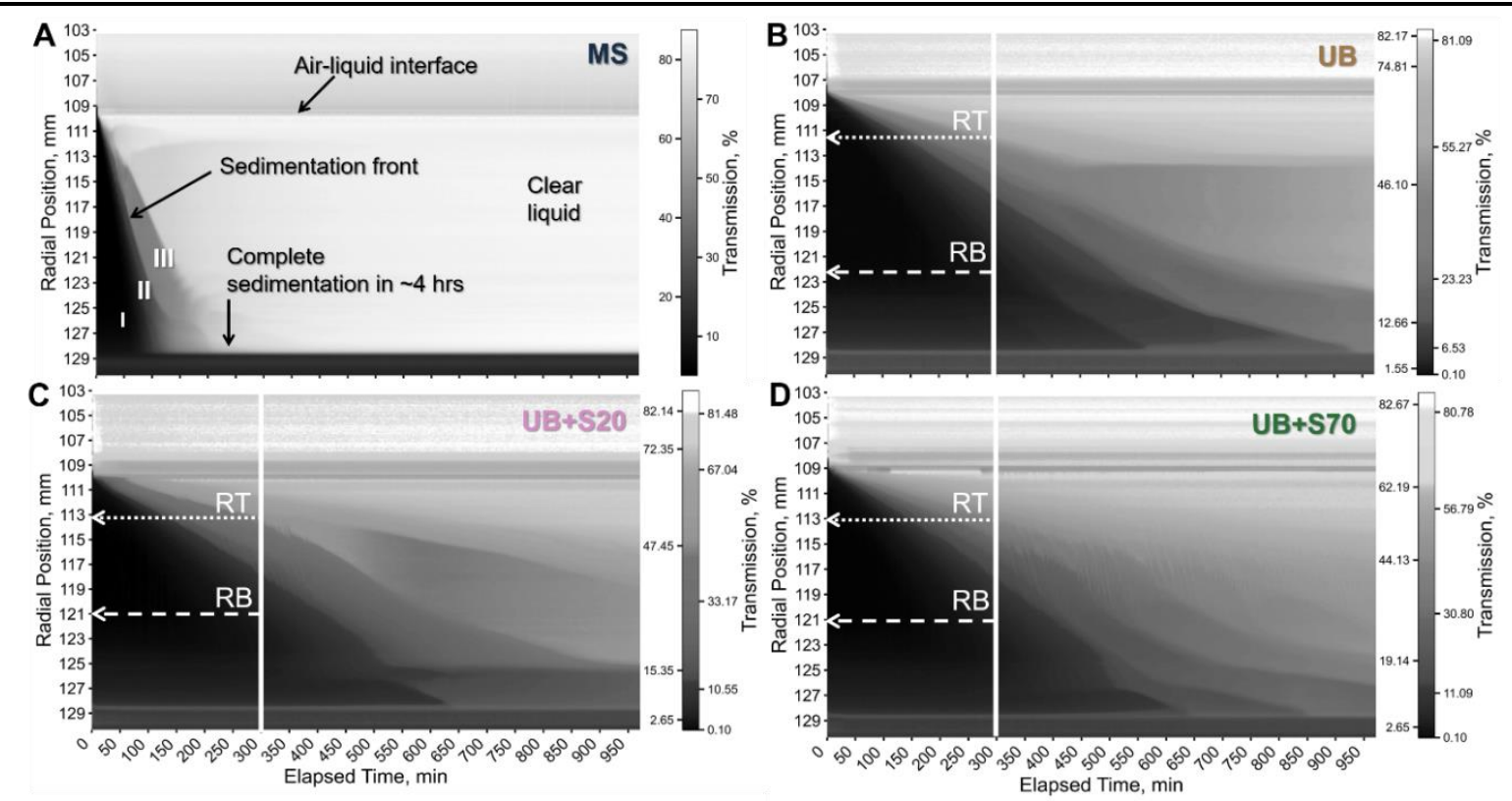

Figure 4: (A) Transmittogram for catalyst ink sample MS. The air-liquid interface and sedimentation front are marked by a black arrow. Three aggregates / agglomerates were clearly observed settling at different speeds resulting in complete sedimentation within 4 hours. Transmittograms for ink samples (B) UB, (C) UB+S20 and (D) UB+S70 respectively. Quantilebased mapping was used for constructing transmittograms for better visualization. The radial positions for which sedimentation rates of topmost (RT) and bottommost (RB) assemblages were calculated are indicated by white lines, while corresponding transmission values are provided as part of the color code. The distance traveled from the start of the experiment (i.e., at the meniscus) to $\mathrm{RB}$ or $\mathrm{RT}$ is used to evaluate the settling rates.

Similarly, representative transmittograms are plotted for inks prepared by ultrasonic bath and probe sonication, as shown in Figure 4B-D. It is evident that complete sedimentation did not take place for the ink samples $\mathrm{UB}, \mathrm{UB}+\mathrm{S} 20$ and $\mathrm{UB}+\mathrm{S} 70$. This confirms the observation made using the fingerprints as well. Gradients in the transmission values and distinct boundaries show the 
presence of several assemblages in the ink samples. Compared to sample MS, there is clear breaking of large assemblages in the presence of probe sonication, which are well-dispersed over long times. Hence qualitative visual confirmation using space-time sedimentation maps reveals several assemblages. Some assemblages sediment much faster, while some remain dispersed even until the end of the experiment. Defining primary carbon particles, which are prenucleating structures, aggregates of primary particles, agglomerates of primary particles as well as agglomerates of primary aggregates are not binary (yes or no) outcomes.

The different sedimentation fronts can be tracked, and the sedimentation times of each assemblage can be evaluated. In addition to the visualization of the settling behavior, transmittograms also allow to calculate the settling rate. For a uniform comparison across the three samples (UB, $\mathrm{UB}+\mathrm{S} 20, \mathrm{UB}+\mathrm{S} 70$ ), the motion of sedimentation fronts is tracked from the start of the experiment to $300 \mathrm{~min}$. The bottommost and the topmost assemblages and the corresponding radial positions RB (radial bottom, dashed arrow) and RT (radial top, dotted arrow) were considered as annotated in Figure 4B-D. RB and RT mark the distance traveled by respective assemblages from the meniscus in 300 mins. Since the ink processed by the magnetic stirrer settled before $300 \mathrm{~min}$, it was excluded from this calculation. Certainly, transmittograms for samples other than MS show the presence of several assemblages whose settling rates can be quantified and compared, but for simplicity, only two were chosen to demonstrate the potential of the technique. Comparing the settling rates from meniscus (at time $=0 \mathrm{~min}$ ) to the marked radial position (at time $=300 \mathrm{~min}$ ) for the bottommost assemblage in the transmittograms for different samples, ink sample UB has the highest rate of $6.82 \mathrm{~cm} /$ day, followed by ink sample $S 70$ with the rate of $6 \mathrm{~cm} /$ day. Ink sample $\mathrm{UB}+\mathrm{S} 20$ settles at the slowest rate of $5.28 \mathrm{~cm} /$ day. Similarly, settling rates are compared for the topmost assemblage in the transmittograms for the three ink samples. As seen in the 
transmittograms, the topmost assemblage is the one that is constituted by smaller sized agglomerates. For $\mathrm{UB}, \mathrm{UB}+\mathrm{S} 20, \mathrm{UB}+\mathrm{S} 70$, the settling rates are $1.82,1.58$, and $1.92 \mathrm{~cm} /$ day, respectively. Comparing the settling rates for assemblages that settle the fastest vs. which settle the slowest indicates several observable trends. Firstly, sample UB+S20 has the slowest relative settling rate for both topmost and bottommost assemblage. Secondly, while UB has the fastest settling bottommost assemblage, $\mathrm{UB}+\mathrm{S} 70$ has the fastest settling topmost assemblage. From this, in line with findings of Retamal Marín et al. [61] on the re-agglomeration of silica particles at high-intensity sonication, we can carefully speculate that the energy input during the preparation of sample $\mathrm{UB}+\mathrm{S} 70$ potentially leads to re-agglomeration of broken assemblages, which settled quickly.

The results of this section establish transmittograms as an effective way to compare, contrast and study the dispersion state. Sample MS is, without doubt, the sample which is not dispersed properly and hence settles completely very fast. Remarkably, the other three samples (UB, UB+S20, $\mathrm{UB}+\mathrm{S} 70$ ), show evidence of incomplete settling, even after being under accelerating centrifugal force for three-quarters of a day. All these samples show the presence of multiple assemblages that are stabilized in the solvent and settle slowly. Tracking sedimentation fronts for these samples hints at some assemblages settling faster compared to others. As a quantitative evaluation of the dynamics of changes in colloidal properties during sedimentation is largely unavailable for complex dispersions, such analyses can become a door-opener for improved understanding. The present results from transmittograms are significant in at least three major aspects. First, a transmittogram enables us to distill information on the colloidal migration of particles. Additionally, it also provides clues on when and how many assemblages are formed. Secondly, the transmittogram directly shows whether complete sedimentation has been achieved. Thirdly, 
settling rates can be evaluated by tracking the sedimentation fronts. This can be done manually (as shown here) or using a machine interface to process images to find edges and boundaries algorithmically. Thus, transmittogram analysis can be a powerful tool for characterizing complex dispersions in their native state, circumventing the need to deconvolve fingerprints to draw meaningful conclusions. Instead, the information-rich spatio-temporal maps provide a facile way to reveal particle migration dynamics.

So far, transmittograms reveal both the (i) initial state of the ink, which is the direct outcome of the shearing forces applied during processing, and (ii) successive state transformations under centrifugal action, which may be arising due to several interacting forces. The latter are associated with stabilization and destabilization actions within the dispersion and possible layering due to thermal instabilities. What is clearly missing from transmittograms is a way to quantify them and the need for information beyond the dispersion state to select the ink preparation method most appropriate for the required stability characteristics. The next section, therefore, moves on to discuss how to quantify dispersion stability using the measured optical transmission.

\subsection{Dispersion stability}

\subsubsection{Stability trajectory}

The transmission profiles captured with $\mathrm{AC}$ were used to calculate $\mathrm{S}$ scores (see Equation 1, 2, and 3). For each profile, an S score value was calculated. Together, the array of S scores plotted over time as a line chart yields a stability trajectory. Figure 5A and 5C show trajectories for ink samples MS and UB+S20, respectively. It should be noted that both samples were centrifuged for 16 hours. Sample MS clearly shows complete settling in about 4 hours. Thus, to provide a granular look at the time duration, which indicates particle migration, only the first $\sim 8$ hours of data are 
shown. The trace describes the evolving stability characteristics of the samples. Corresponding transmittograms (see Figure 5B and 5D) are plotted below with shared time axes.

Recollecting that the time point corresponding to the valleys in the stability trajectory can be mapped to complete settling of a distinct assemblage. This can be seen while comparing Figures $5 \mathrm{~A}$ and $5 \mathrm{~B}$, where the largest assemblage settled completely. For simple particle systems, the location of valleys is very characteristic to complete settling times. This can be illustrated using model particle systems (bimodal and 4-modal silica in water) in supporting Figures S2A and S2B, respectively. However, it has to be noted that for complex catalyst inks, this may not always be easily visible as sedimentation fronts are lesser defined due to pronounced dispersity.

Since a higher value of S score indicates greater instability, the trajectory of sample MS quickly ascends into greater instability, compared to $\mathrm{UB}+\mathrm{S} 20$. In the first $50 \mathrm{~min}$, the $\mathrm{S}$ score for MS increases four times. In contrast, $\mathrm{UB}+\mathrm{S} 20$ sees no increase in the S score value. In other words, for MS trajectory, to reach an S score value of 0.4, it takes about $50 \mathrm{~min}$, whereas for UB+S20 trajectory it takes about $375 \mathrm{~min}$. Evidently, the rate of increase of the trajectory shows how fast the sample develops a state of higher disorder at a certain measurement time due to particle migration i.e., how fast separation proceeds. This can be easily confirmed by looking at the juxtaposed transmittograms. Clearly, MS shows settling at a faster rate, compared to UB+S20.

The ascending zone indicates how quickly the dispersed phase migrates, such that the largest particles (which will naturally settle sooner than smaller ones) in the dispersion have settled. The descending zone of the trajectory indicates how soon the dispersion clarifies to obtain a pure continuous phase. Noteworthy, these interpretations are in line with the conclusions drawn from transmittograms. The ascent reaches a point where the S score exhibits its maximum value of 1.04 and 1.16 for MS and UB+S20, respectively. The corresponding BSTs are 105 and 805 min (marked 
by a blue vertical line joining the peak to the x-axis). Recalling that the BST corresponds to the highest $\mathrm{S}$ score, i.e., the highest MAD value of the z-normalized profiles. The transmittogram shows a distribution of lower transmission values to the left of this time point (darker shades). Conversely, higher transmission values are prevalent to the right of this time point (lighter shades). Beyond the BST, the trajectory plummets to a value of 0.2 for MS, and 0.75 for $\mathrm{UB}+\mathrm{S} 20$. The value of the $\mathrm{S}$ score is persistently low beyond this time point as the transmittogram confirms that only the continuous phase is present, and the dispersed phase has settled. Together, the plots in Figure 5 allow us to draw conclusions from the transmittogram on the dispersion characteristics and substantiate the observations from the stability trajectory, and vice versa. 


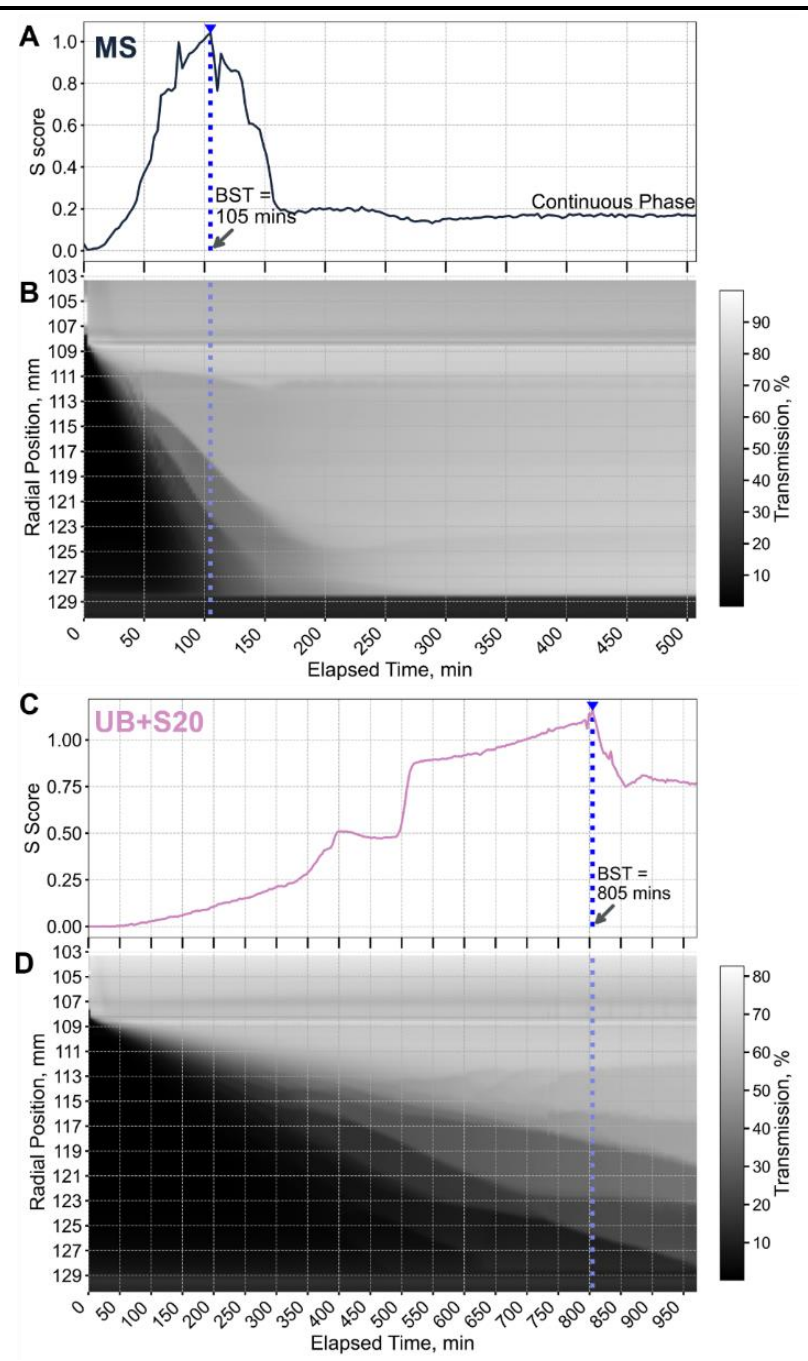

Figure 5: (A) Stability trajectory and (B) transmittogram of ink sample MS. The bulk sedimentation is achieved in about $105 \mathrm{~min}$ as indicated by a blue vertical dotted line. The continuous phase is clarified within 300 min as shown by the lower S score line. (C) Stability trajectory and (D) transmittograms of ink sample UB+S20. The BST of 805 min is indicated by a blue vertical dotted line.

One considerable advantage in employing stability trajectories is the ability to compare them for samples that may not have been captured in the same experimental run. As long as the centrifugal acceleration is constant, experiments of different duration can also be compared. For instance, the 
trajectory for an ink sample run for a duration of $\sim 16$ hours only (see supporting Figure S3) does not change when only a part of the total time is considered for evaluation, i.e., $\sim 5$ hours.

Furthermore, we note that a time point corresponding to the maximum S score for a shorter period trajectory may not correspond to the true BST. This is because such peaks can be local maxima. A more extended period experiment at the same RCF may reveal a rising trajectory that peaks at an ensuing time point, revealing the global maximum, which would be the true BST. On encountering such a case, the user can examine the transmittogram and the shape of the trajectory and decide if BST comparisons shall be made with additional (longer time or higher RCF) experiments. This is one of the main advantages of the trajectory and transmittogram, together assisting in unraveling the state and stability of the system.

The full strength of S score and the trajectory, however, becomes clear when samples are directly compared with each other. Figure 6 shows representative stability trajectories of the four ink samples - MS (panel A), UB (panel B), UB+S20 (panel C), and UB+S70 (panel D), comparing the time-resolved response of the dispersion state. These are the same samples for which transmittograms are seen in Figure 4. For trajectories of three different samples for each processing method, we refer to supporting Figure S4. It becomes clear that the evolution of S score is highly reproducible from one experiment to another and that changes between differently processed samples are obvious. The maxima for the four trajectories are shown with a blue inverted triangle. The dotted blue vertical line joins the maxima to the time axis to indicate the BST.

It immediately becomes clear that $\mathrm{S}$ score values are the highest for $\mathrm{UB}+\mathrm{S} 20$ and lowest for MS. The latter is the case because only the continuous phase is present, whereas the former indicates the widespread presence of dispersed particles. After UB+S20, sample UB+S70 also shows a larger S score value at the end, which is indicative of the presence of many assemblages. 
Interestingly, the S scores around the zeroth time are temporarily high, as the sample undergoes transient but severe upheaval due to a jerk motion at the start of rotor motion. Together, these observations corroborate with what we can see in the transmittograms (see Figure 4), however, at the clear advantage of significant data reduction. This is getting increasingly important when numerous datasets are to be analyzed, which is the only way to ultimately replace prevailing empiricism in formulation (science) by knowledge-based design.

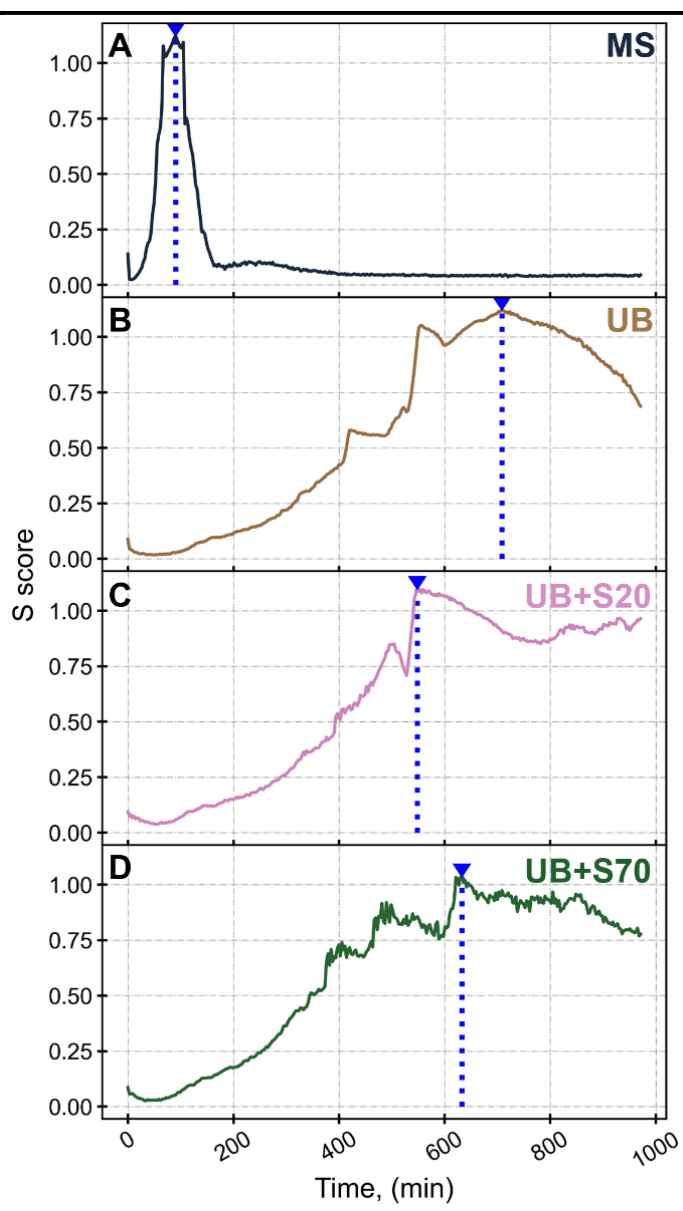

Figure 6. Stability trajectory for catalyst ink samples (A) MS (dark blue line), (B) UB (brown line), (C) UB+S20 (magenta line) and (D) UB+S70 (dark green line), respectively. Corresponding blue dotted lines and inverted triangles show the global maxima indicating the BST. 
In Figure 6 the discriminating feature of MS trajectory (panel A) is that it rises, reaches a peak, descends, and then remains constant until the end. This differs entirely from the trajectory of UB+S20 (panel C), which first ascends gradually, dips briefly ( 500 min), increases sharply ( 540 $\min )$ before it peaks ( $\sim 560 \mathrm{~min})$, and then drops progressively and goes into a plateau phase. Thus, the shape of a stability trajectory is unique to the dispersion sample it represents.

Herein, the BST is a very useful parameter, which is explanatory for the behavior of the trajectory. In this study, the concept of BST takes prominence as inks of three of the four processing methods do not entirely sediment after 16 hours of experimental run time. Hence time for complete sedimentation is inappropriate for comparing the different ink samples. Having seen how trajectories are descriptive of the evolving dispersion stability, the following section relates the BST parameter for all the measured samples.

\subsubsection{Comparison of bulk sedimentation times}

In the following, the BSTs for the four processing methods are compared. As mentioned in the experimental section, three samples for each of the four dispersion methods were prepared to assure the experimental reproducibility. Then, each of the three samples was measured in triplicate to check the repeatability of the measurement using the photocentrifuge. Thus, nine data points were obtained for each dispersion procedure. Figure 7 shows the BSTs on the $y$-axis and the processing method on the $x$-axis. The hollow points of the same shape (square, or circle, or triangle) represent the triplicate measurements of the same ink dispersion. Filled diamonds are the means for the triplicates of each of the three different ink dispersions. The horizontal-colored lines show the mean for all the measurements, and the error bar shows the standard error of the means. Sample MS has exceptionally low BSTs ( 100 min). The mean BSTs for samples UB and UB+S20 
are similar, with $\mathrm{UB}+\mathrm{S} 20$ being marginally higher than UB. It is striking that the mean BST for ink sample $\mathrm{UB}+\mathrm{S} 70$ is lower.

As discussed previously, studies report that high energy input resulting from larger oscillation amplitudes often leads to re-agglomeration of the broken assemblages and induce various physicochemical alterations on the surface of the constituents of dispersion [62-64]. The deagglomeration of nanoparticle suspensions occurs to a certain vibrational amplitude, as explained by Nguyen et al. [65]. Hence, operating beyond the optimum amplitude or energy input results in insignificant size reduction and sometimes even a re-agglomeration. The lower BST indicates lower stability of sample UB+S70 compared to that of samples UB and UB+S20. Although more systematic studies clearly beyond the scope of this work would be needed, this could be attributed to the re-agglomeration of particles as a result of an increased number of effective collisions at a higher amplitude. At the same time, it could also be caused by the rupture of Nafion ${ }^{\circledR}$ due to cavitation and shock waves, destabilizing the ink structure as suggested in the literature [66]. 


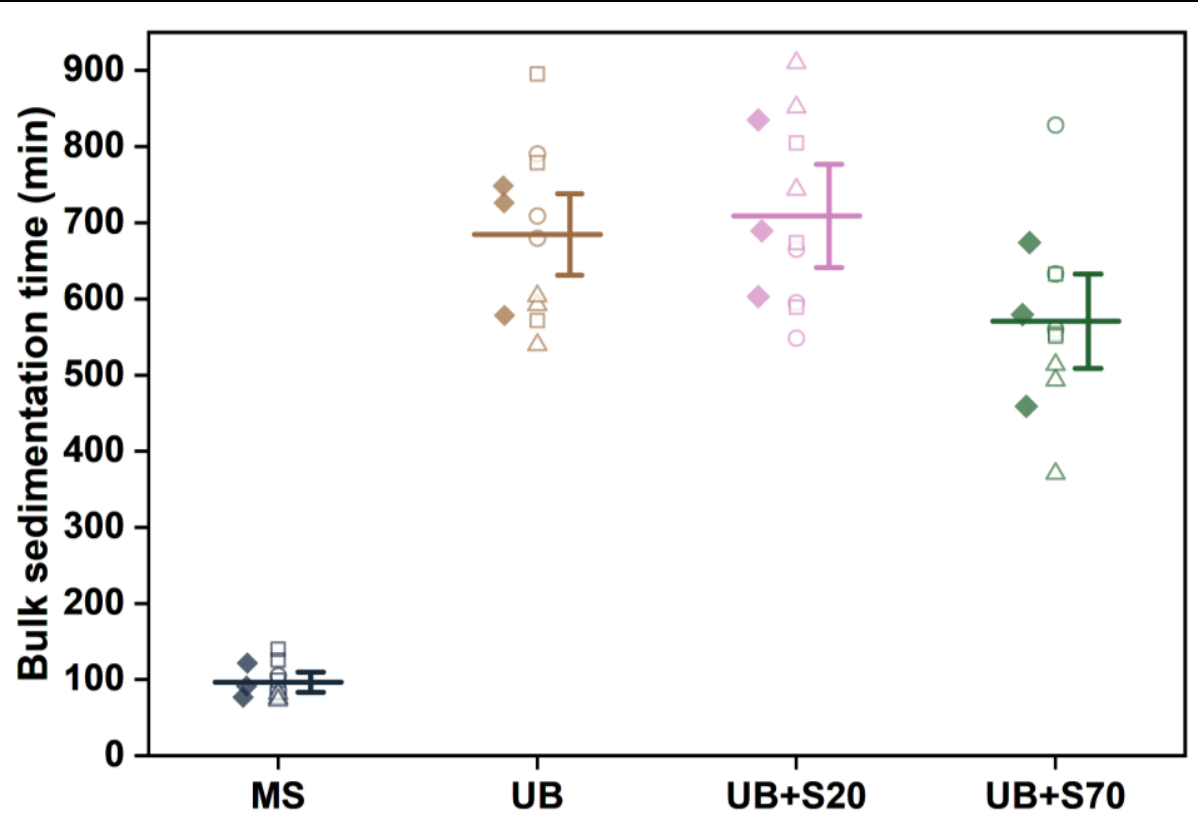

Figure 7. BSTs for catalyst ink samples MS (dark blue), UB (brown), UB+S20 (magenta) and $\mathrm{UB}+\mathrm{S} 70$ (dark green) respectively. Hollow squares, hollow rectangles and hollow triangles show triplicate measurements of the corresponding ink preparation method. The filled diamonds show the means of triplicates for sedimentation times of three different inks. The mean of all measurements is shown by the horizontal-colored lines along with the standard error of the means.

At this point, it needs to be mentioned that quantitative appraisal of stability (or the lack of it) is also possible by calculation of instability index, provided by SepView@. Instability index allows assessment of the stability of samples by comparing the values at any user-defined time point. In Figure S5, mean instability indices for three different inks are plotted with time for the four processing methods. However, it can be seen that instability indices make no attempt to properly describe the stability characteristics such as individual assemblage settling times due to a monotonically increasing curve. In comparison, the outcome of studying the stability trajectory 
emerges to provide descriptive and deductive stability parameters, at least for the study of catalyst inks.

In summary, a unified approach to examine the duality of state and stability in the context of dispersions at technically relevant application concentrations is described. The tools presented not only provide rich insights but also quantify them for objective comparisons of complex interactions of multiple entities in a dispersion. To finally close a first process-structure-property relationship [67], we now move to examine the effect of different catalyst ink processing methods on its rheology (handling property and solution-coating performance).

\subsection{Handling properties using rheological characterization}

Rheological testing assists in process optimization and monitoring quality control. Noteworthy, it also allows to connect the disperse state and stability of the system under investigation with handling properties [68]. The effects of the four processing methods on the ink rheology were investigated. Figure 8 displays the viscosity curves of the inks with identical composition and concentration. Viscosity data recorded from $0.1 \mathrm{~s}^{-1}$ to $10 \mathrm{~s}^{-1}$ show relatively large variation due to torques below the lower limit of the rheometer, and thus data from shear rate $10 \mathrm{~s}^{-1}$ to $1000 \mathrm{~s}^{-1}$ are shown in the figure. Different markers represent the mean value of three measurements made on three separately prepared ink samples to assure the experimental reproducibility and the measurement repeatability. The different colored ribbons are made by connecting the error bars, which represent the standard deviations of the three measurements.

All ink samples demonstrate nearly Newtonian behavior between shear rates of 10 to $1000 \mathrm{~s}^{-1}$. Ink sample MS shows the highest mean viscosity. In case of inks prepared by ultrasonic treatment, sample UB shows slightly lower mean viscosity compared to the mean viscosity of sample MS; 
this difference ranges from $0.57 \%\left(1000 \mathrm{~s}^{-1}\right)$ to $2 \%\left(10 \mathrm{~s}^{-1}\right)$. Remarkably, UB inks show a large variability between three independently prepared inks. This variability is so large that it completely overlaps with the results of MS samples. When the ink prepared by ultrasonic bath is treated by probe sonication for $10 \mathrm{~min}$, like in case of samples $\mathrm{UB}+\mathrm{S} 20$ and $\mathrm{UB}+\mathrm{S} 70$, the viscosity is further reduced. When compared with the mean viscosity of sample MS, samples $\mathrm{UB}+\mathrm{S} 20$ and $\mathrm{UB}+\mathrm{S} 70$ exhibit a reduction of $9 \%$ to $14 \%$ and $18 \%$ to $22 \%$ in mean viscosities over the shear rate range, respectively. Although the mean viscosities for $\mathrm{UB}+\mathrm{S} 70$ samples are the lowest, they show very large variation (similar to UB) over three independently prepared ink samples. It must be noted that the time tests show a linear viscosity increase of maximum $5 \%$ after $3600 \mathrm{~s}$ (data not shown here). The viscosity increase due to drying effects after about $360 \mathrm{~s}$ (sample loading + thermal equilibration + measurement) may thus account for an overestimation of up to $0.5 \%$ for the last data point recorded, assuming a linear interrelation.

From the perspective of the production process of a CCM, both viscosity being too high (as in the case of ink MS) and too low (as in the case of UB+S70) might lead to clogging of a nozzle while spraying or inkjet printing. Both processes involve high shear rates and might thus lead to shearthickening for higher viscosity samples (shear-thickening with high solid loading) or the formation of vortices (non-laminar flow conditions) more likely for low viscosity samples. Very low viscosity might additionally lead to satellite droplets. Thus, an optimum viscosity would be desirable in a given working range. Herein, the results for $\mathrm{UB}+\mathrm{S} 20$ show that such an ink dispersion would be the most favorable.

Starting with the extrema, ink sample MS shows the highest mean viscosity. This is consistent with the findings from stability analysis, which show the lowest BST for sample MS, hinting at the least stable ink. Another notable observation is that UB+S70 shows the lowest mean viscosity. 
Generally, a decrease in viscosity may be attributed to a greater extent of stabilization due to the breakage of aggregates and agglomerates. However, this must be interpreted with care, as evidence from stability analysis brings to light the relatively faster settling of particles. Figure 7 clearly depicted a lower BST of ink sample $\mathrm{UB}+\mathrm{S} 70$ in comparison to samples UB and $\mathrm{UB}+\mathrm{S} 20$. This decrease in BST indicates faster settling of the majority of the particles and hence indicating lower stability. Lower viscosity for UB+S70 can be postulated due to (i) rupturing of ionomer chains and (ii) propensity of particles to re-agglomerate. Thus, the most likely explanation for reduced viscosity of UB+S70 is the degradation of polymers (in this case, Nafion $®$ ) by applying higher ultrasonic energy $[63,69]$. Similar observations have been discussed in a few reports. For example, Adamski et al. [70] systematically studied the effects of power ultrasound on Nafion® dispersion properties. The results showed a significant reduction in the viscosity of Nafion ${ }^{\circledR}$ on ultrasonication. This was alluded to the degradation of polymer chains due to the combination of hydrodynamic cavitation and mechanical shearing forces. In contrast, only $\sim 1 \%$ decrease in viscosity was observed under stirring conditions of 1000 RPM for a day. Safronova et al. [66] also reported a reduction of viscosity of Nafion ${ }^{\circledR}$ caused by the rupture of macromolecule chains upon ultrasonic treatment. Further, systematic studies to confirm the degradation of Nafion ${ }^{\circledR}$ at higher oscillation amplitudes and their effects on ink properties would be needed. Nevertheless, this study shows that optimization of the dispersion procedure itself may supersede the need to introduce rheological additives, which might be used to tailor the flowability, thus avoiding possible negative effects on the performance of the resulting electrode.

The final section of this study thus brings to light intriguing relations between the stability and rheology of catalyst ink. It also shows how an independent approach to colloidal stability potentially allows to better understand the fluid flow of disperse systems. The latter is literally the 
integral response of the system to all interactions present when the ink is exposed to external stress and thus often challenging to interpret. The results motivate the premise for quantitatively assessing dispersion stability in combination with handling properties for a knowledge-based approach to catalyst ink formulation.

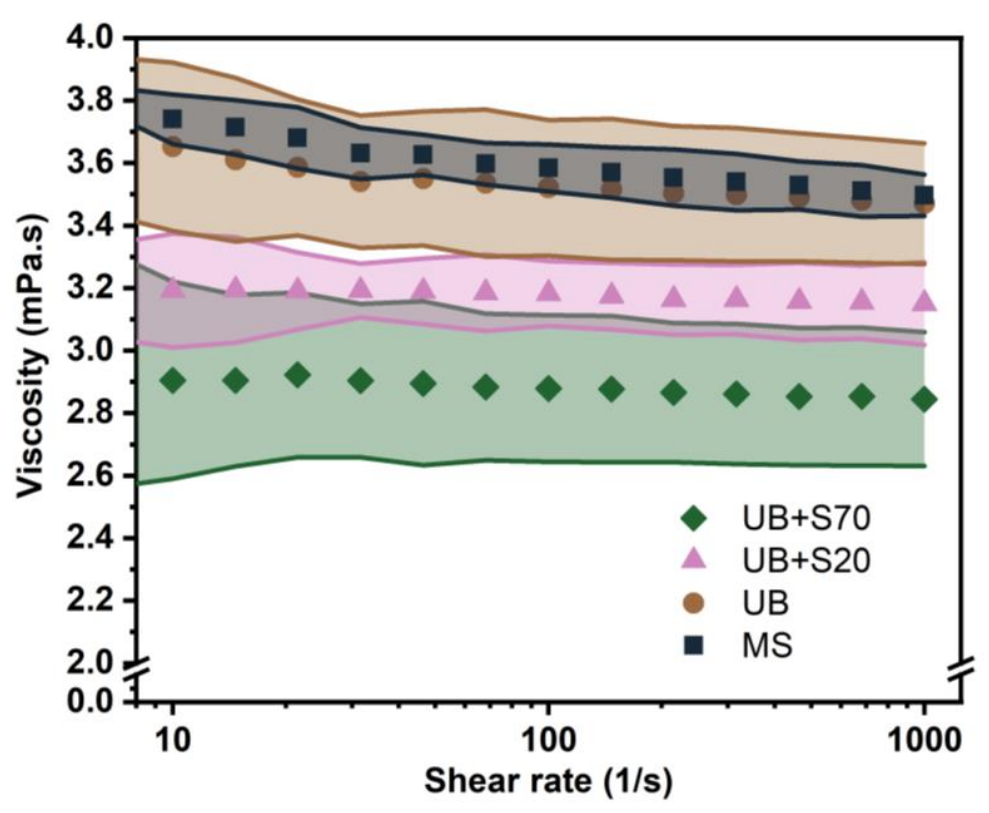

Figure 8. Viscosity-shear rate curves for catalyst ink sample MS (dark blue square), UB (brown circle), UB+S20 (magenta triangle) and UB+S70 (dark green diamond) respectively. Corresponding colored ribbons are made by joining the standard deviations of three measurements.

\section{Conclusion}

Studies on model systems are reported in abundance; however, they contribute to industrial problems in a limited way only. This work is a step forward in the direction of approaching relevant formulation conditions, i.e., i) highest degree of polydispersity, ii) multi-material dispersed phase iii) mixtures of liquids as the continuous phase, and iv) high, technical concentrations. On one 
side, characterization tools and techniques (methods), and on the other side, the improvement of ink dispersion stability and suitability for layer preparation, in the end for enhanced PEMFC catalyst layer performance (application), together form two sides of the same coin. Through this work, we investigate 'both sides of the coin' to elucidate process-structure and structure-property relationships. On the methods side of the coin, we demonstrate the utility of a photocentrifuge as a characterization tool for complex fuel cell catalyst ink dispersions. As a step forward, we show how the state and stability of the dispersion can be evaluated concurrently. Using transmittograms, direct visual cues provide a dossier of presence and number of assemblages. By calculating $S$ scores, we introduce a new concept of stability trajectory. The latter is derived from the presentation of S scores as a time series, which allows the tracking of dynamics of the particle migration. Together these two procedures of analyses can be easily integrated with typical measurement and assessment workflows. On the application side of the coin, we put to use the developed techniques and present an approach to systematically study the effect of ink dispersion preparation methods. We try to extract process-structure-property relations by preparing ink dispersions using four methods, which are of industrial importance. The stability of the dispersion, formation of different assemblages is then investigated using AC. We see a clear effect of the ink processing on stability and rheology. For quality control purposes in production, it is important to consider the effects of rheology and stability and find an optimum balance between them. Considering dispersion stability, transmittogram analysis and stability trajectory show that $\mathrm{UB}+\mathrm{S} 20$ has more optimum dispersion stability. Also, rheology results show the lowest viscosity of UB+S70, i.e., the sample prepared with the highest energy input, rather likely because of degradation of ionomer. Future outcomes of this approach may include printing or spraying the well-dispersed ink to form the CL, around which a CCM and an MEA can be constructed. Hence 
the fuel cell performance can be measured and correlated to the processing method, stability, and rheology of the catalyst ink.

\section{Acknowledgments}

SB gratefully acknowledges a scholarship from the IMPRS-SurMat doctoral program. Special thanks are due to the Interdisciplinary Center for Analytics on the Nanoscale (ICAN, Dr. Markus Heidelmann) for TEM measurements. SB, DS, and VP acknowledge the financial support from the Federal Ministry for Economic Affairs and Energy (WIPANO funding scheme, project number 03TN0013A). SK and VP acknowledge the support by the Federal Ministry of Economics and Technology, Germany (funding number 30EWBG/2, "Dreidimensionale (3D) Gestaltung von Katalysatorschichten einer PEM-Brennstoffzelle im Inkjetdruck für eine hocheffiziente Ausnutzung des Platinkatalysators (3D-KAT)").

\section{References}

[1] Y. Wang, K. S. Chen, J. Mishler, S. C. Cho, X. C. Adroher, A review of polymer electrolyte membrane fuel cells: Technology, applications, and needs on fundamental research, Appl. Energy. 88 (2011) 981-1007. https://doi.org/10.1016/j.apenergy.2010.09.030.

[2] A. Albarbar, M. Alrweq, Proton Exchange Membrane Fuel Cells, in: Prot. Exch. Membr. Fuel Cells, Springer, 2018: pp. 9-29.

[3] R. N. Bonifácio, J. O. A. Paschoal, M. Linardi, R. Cuenca, Catalyst layer optimization by surface tension control during ink formulation of membrane electrode assemblies in proton exchange membrane fuel cell, J. Power Sources. 196 (2011) 4680-4685. https://doi.org/10.1016/j.jpowsour.2011.01.010. 
[4] J. C. Park, S. H. Park, M. W. Chung, C. H. Choi, B. K. Kho, S. I. Woo, Optimization of catalyst layer composition for PEMFC using graphene-based oxygen reduction reaction

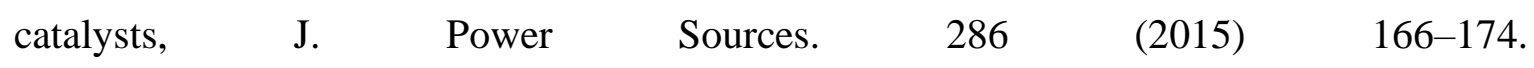
https://doi.org/10.1016/j.jpowsour.2015.03.137.

[5] A. Ganesan, M. Narayanasamy, Ultra-low loading of platinum in proton exchange membrane-based fuel cells: a brief review, Mater. Renew. Sustain. Energy. 8 (2019). https://doi.org/10.1007/s40243-019-0156-x.

[6] F. C. Cetinbas, S. G. Advani, A. K. Prasad, Investigation of a polymer electrolyte membrane fuel cell catalyst layer with bidirectionally-graded composition, J. Power Sources. 270 (2014) 594-602. https://doi.org/10.1016/j.jpowsour.2014.07.148.

[7] Y. Zhang, A. Smirnova, A. Verma, R. Pitchumani, Design of a proton exchange membrane ( PEM ) fuel cell with variable catalyst loading, J. Power Sources. 291 (2015) 46-57. https://doi.org/10.1016/j.jpowsour.2015.05.002.

[8] U.S. Department of Energy, DOE Technical Targets for Polymer Electrolyte Membrane Fuel Cell Components, (n.d.). https://www.energy.gov/eere/fuelcells/doe-technical-targetspolymer-electrolyte-membrane-fuel-cell-components (accessed September 21, 2020).

[9] Z. Xie, X. Zhao, M. Adachi, Z. Shi, T. Mashio, A. Ohma, K. Shinohara, S. Holdcroft, T. Navessin, Fuel cell cathode catalyst layers from “green” catalyst inks, Energy Environ. Sci. 1 (2008) 184-193. https://doi.org/10.1039/b808613n.

[10] K. Malek, T. Mashio, M. Eikerling, Microstructure of Catalyst Layers in PEM Fuel Cells Redefined: A Computational Approach, Electrocatalysis. 2 (2011) 141-157. https://doi.org/10.1007/s12678-011-0047-0. 
[11] M. Bredol, A. Szydło, I. Radev, W. Philippi, R. Bartholomäus, V. Peinecke, A. Heinzel, How the colloid chemistry of precursor electrocatalyst dispersions is related to the polymer electrolyte membrane fuel cell performance, J. Power Sources. 402 (2018) 15-23. https://doi.org/10.1016/j.jpowsour.2018.09.005.

[12] C. R. Randall, S. C. Decaluwe, Physically Based Modeling of PEMFC Cathode Catalyst Layers : Effective Microstructure and Ionomer Structure - Property Relationship Impacts, J. Electrochem. Energy Convers. Storage. $17 \quad$ (2020) 1-10. https://doi.org/10.1115/1.4046417.

[13] Y. Li, D. Xiong, Y. Liu, M. Liu, J. Liu, C. Liang, C. Li, Correlation between electrochemical performance degradation and catalyst structural parameters on polymer electrolyte membrane fuel cell, Nanotechnol. Rev. 8 (2019) 493-502.

[14] S. Takahashi, T. Mashio, N. Horibe, K. Akizuki, A. Ohma, Analysis of the Microstructure Formation Process and Its Influence on the Performance of Polymer Electrolyte Fuel-Cell Catalyst Layers, ChemElectroChem. $2 \quad$ (2015) 1560-1567. https://doi.org/10.1002/celc.201500131.

[15] M. B. Sassin, Y. Garsany, B. D. Gould, K. E. Swider-Lyons, Fabrication Method for Laboratory-Scale High-Performance Membrane Electrode Assemblies for Fuel Cells, Anal. Chem. 89 (2017) 511-518. https://doi.org/10.1021/acs.analchem.6b03005.

[16] M. Wang, J. H. Park, S. Kabir, K. C. Neyerlin, N. N. Kariuki, H. Lv, V. R. Stamenkovic, D. J. Myers, M. Ulsh, S. A. Mauger, Impact of Catalyst Ink Dispersing Methodology on Fuel Cell Performance Using in-Situ X-ray Scattering, ACS Appl. Energy Mater. 2 (2019) 6417-6427. https://doi.org/10.1021/acsaem.9b01037. 
[17] D. C. Huang, P. J. Yu, F. J. Liu, S. L. Huang, K. L. Hsueh, Y. C. Chen, C. H. Wu, W. C. Chang, F. H. Tsau, Effect of dispersion solvent in catalyst ink on proton exchange membrane fuel cell performance, Int. J. Electrochem. Sci. 6 (2011) 2551-2565.

[18] P. Gallo Stampino, C. Cristiani, G. Dotelli, L. Omati, L. Zampori, R. Pelosato, M. Guilizzoni, Effect of different substrates, inks composition and rheology on coating deposition of microporous layer (MPL) for PEM-FCs, Catal. Today. 147 (2009) 30-35. https://doi.org/10.1016/j.cattod.2009.07.023.

[19] E. Hoffmann, S. Zhang, M. Thoma, C. Damm, W. Peukert, Formulation of carbon blackionomer dispersions for thin film formation in fuel cells, Particuology. 44 (2019) 7-21. https://doi.org/10.1016/j.partic.2018.08.001.

[20] S. A. Mauger, K. C. Neyerlin, A. C. Yang-Neyerlin, K. L. More, M. Ulsh, Gravure coating for roll-to-roll manufacturing of proton-exchange-membrane fuel cell catalyst layers, J. Electrochem. Soc. 165 (2018) F1012-F1018. https://doi.org/10.1149/2.0091813jes.

[21] S. A. Mauger, Material-process-performance relationships for roll-to-roll coated PEM electrodes, US DOE 2017 Hydrog. Fuel Cells Annu. Merit Reivew. (2017).

[22] M.B. Dixit, B.A. Harkey, F. Shen, K.B. Hatzell, Catalyst layer ink interactions that affect $\begin{array}{llllll}\text { coatability, } & \text { J. } & \text { Electrochem. } & \text { Soc. } & 165 & \text { (2018) }\end{array}$ https://doi.org/10.1149/2.0191805jes.

[23] S. Shukla, K. Domican, K. Karan, S. Bhattacharjee, M. Secanell, Analysis of Low Platinum Loading Thin Polymer Electrolyte Fuel Cell Electrodes Prepared by Inkjet Printing Electrochimica Acta Analysis of Low Platinum Loading Thin Polymer Electrolyte Fuel Cell Electrodes Prepared by Inkjet Printing, Electrochim. Acta. 156 (2015) 289-300. 
https://doi.org/10.1016/j.electacta.2015.01.028.

[24] A. Lesch, F. Cortés-Salazar, V. C. Bassetto, V. Amstutz, H. H. Girault, Inkjet printing meets electrochemical energy conversion, Chimia (Aarau). 69 (2015) 284-289. https://doi.org/10.2533/chimia.2015.284.

[25] S. Holdcroft, Fuel cell catalyst layers: A polymer science perspective, Chem. Mater. 26 (2014) 381-393. https://doi.org/10.1021/cm401445h.

[26] D. Walter, Primary particles--agglomerates--aggregates, in: Nanomaterials, Wiley Online Library, 2013: pp. 9-24.

[27] B. Millington, V. Whipple, B. G. Pollet, A novel method for preparing proton exchange membrane fuel cell electrodes by the ultrasonic-spray technique, J. Power Sources. 196 (2011) 8500-8508. https://doi.org/10.1016/j.jpowsour.2011.06.024.

[28] S. Shukla, S. Bhattacharjee, A.Z. Weber, M. Secanell, Experimental and Theoretical Analysis of Ink Dispersion Stability for Polymer Electrolyte Fuel Cell Applications, J. Electrochem. Soc. 164 (2017) F600-F609. https://doi.org/10.1149/2.0961706jes.

[29] A. Gluesen, S.D. Mueller, Martin, Slot-Die Coating : A New Preparation Method for Direct Methanol Fuel Cells Catalyst Layers, J. Fuel Cell Sci. Technol. 10 (2016) 1-4. https://doi.org/10.1115/1.4024607.

[30] R. Balzarotti, S. Latorrata, P. G. Stampino, C. Cristiani, G. Dotelli, Development and Characterization of Non-Conventional Micro-Porous Layers for PEM Fuel Cells, Energies. 8 (2015) 7070-7083. https://doi.org/10.3390/en8077070.

[31] X. Ding, T. A. L. Harris, Review on Penetration and Transport Phenomena in Porous Media 
during Slot Die Coating, J. Polym. Sci. Part B Polym. Phys. 55 (2017) 1669-1680. https://doi.org/10.1002/polb.24307.

[32] T. Soboleva, K. Malek, Z. Xie, T. Navessin, S. Holdcroft, PEMFC catalyst layers: The role of micropores and mesopores on water sorption and fuel cell activity, ACS Appl. Mater. Interfaces. 3 (2011) 1827-1837. https://doi.org/10.1021/am200590w.

[33] S. Khandavalli, J. H. Park, N. N. Kariuki, D. J. Myers, J. J. Stickel, K. Hurst, K. C. Neyerlin, M. Ulsh, S. A. Mauger, Rheological Investigation on the Microstructure of Fuel Cell Catalyst Inks, ACS Appl. Mater. Interfaces. 10 (2018) 43610-43622. https://doi.org/10.1021/acsami.8b15039.

[34] A. Therdthianwong, P. Ekdharmasuit, S. Therdthianwong, Fabrication and performance of membrane electrode assembly prepared by a catalyst-coated membrane method: Effect of solvents used in a catalyst ink mixture, Energy and Fuels. 24 (2010) 1191-1196. https://doi.org/10.1021/ef901105k.

[35] T. T. Ngo, P. H. Su, T. L. Yu, Conformation of Nafion Molecules in Dilute Isopropyl Alcohol / Water Mixture Solutions, in: Proc. 3rd International Workshop on Nanotechnology and Application, Vung Tau, Vietnam, 2011, AMN-005-P.

[36] S. A. Berlinger, B. D. McCloskey, A. Z. Weber, Inherent Acidity of Perfluorosulfonic Acid Ionomer Dispersions and Implications for Ink Aggregation, J. Phys. Chem. B. 122 (2018) 7790-7796. https://doi.org/10.1021/acs.jpcb.8b06493.

[37] S. J. Shin, J. K. Lee, H. Y. Ha, S. A. Hong, H. S. Chun, I. H. Oh, Effect of the catalytic ink preparation method on the performance of polymer electrolyte membrane fuel cells, J. Power Sources. 106 (2002) 146-152. 
[38] T. Mashio, K. Malek, M. Eikerling, A. Ohma, H. Kanesaka, K. Shinohara, Molecular dynamics study of ionomer and water adsorption at carbon support materials, J. Phys. Chem. C. 114 (2010) 13739-13745. https://doi.org/10.1021/jp1034135.

[39] M. Thoma, W. Lin, E. Hoffmann, M. M. Sattes, D. Segets, C. Damm, W. Peukert, Simple and Reliable Method for Studying the Adsorption Behavior of Aquivion Ionomers on Carbon Black Surfaces, Langmuir. $34 \quad$ (2018) 12324-12334. https://doi.org/10.1021/acs.langmuir.8b02726.

[40] B. G. Pollet, The use of ultrasound for the fabrication of fuel cell materials, Int. J. Hydrogen Energy. 35 (2010) 11986-12004. https://doi.org/10.1016/j.jhydene.2010.08.021.

[41] B. G. Pollet, J. T. E. Goh, The importance of ultrasonic parameters in the preparation of fuel cell catalyst inks, Electrochim. Acta. $128 \quad$ (2014) 292-303. https://doi.org/10.1016/j.electacta.2013.09.160.

[42] D. Segets, Analysis of particle size distributions of quantum dots: From theory to application, KONA Powder Part. J. (2016) 48-62. https://doi.org/10.14356/kona.2016012.

[43] C. Ullmann, F. Babick, R. Koeber, M. Stintz, Performance of analytical centrifugation for the particle size analysis of real-world materials, Powder Technol. 319 (2017) 261-270. https://doi.org/10.1016/j.powtec.2017.06.057.

[44] F. Xu, H. Zhang, J. Ilavsky, L. Stanciu, D. Ho, M. J. Justice, H. I. Petrache, J. Xie, Investigation of a catalyst ink dispersion using both ultra-small-angle X-ray scattering and cryogenic TEM, Langmuir. 26 (2010) 19199-19208. https://doi.org/10.1021/la1028228.

[45] B. Mert, Using high pressure microfluidization to improve physical properties and lycopene 
content of ketchup type products, J. Food Eng. 109 (2012) 579-587.

[46] A. Zielińska, C. Martins-Gomes, N. R. Ferreira, A. M. Silva, I. Nowak, E. B. Souto, Antiinflammatory and anti-cancer activity of citral: Optimization of citral-loaded solid lipid nanoparticles (SLN) using experimental factorial design and LUMiSizer®, Int. J. Pharm. 553 (2018) 428-440.

[47] S. B. Barrios, J. F. Petry, C. K. Weiss, C. L. Petzhold, K. Landfester, Polymeric coatings based on acrylic resin latexes from miniemulsion polymerization using hydrocarbon resins as osmotic agents, J. Appl. Polym. Sci. 131 (2014) 1-9. https://doi.org/10.1002/app.40569.

[48] S. Bapat, D. Segets, Sedimentation Dynamics of Colloidal Formulations through Direct Visualization: Implications for Fuel Cell Catalyst Inks, ACS Appl. Nanomater. 3 (2020) 7384-7391. https://doi.org/10.1021/acsanm.0c01467.

[49] T. Detloff, T. Sobisch, D. Lerche, Particle size distribution by space or time dependent extinction profiles obtained by analytical centrifugation (concentrated systems), Powder Technol. 174 (2007) 50-55. https://doi.org/10.1016/j.powtec.2006.10.021.

[50] H. Wang, P. Tan, D. Barona, G. Li, S. Hoe, D. Lechuga-Ballesteros, D. S. Nobes, R. Vehring, Characterization of the suspension stability of pharmaceuticals using a shadowgraphic imaging method, Int. J. Pharm. 548 (2018) 128-138. https://doi.org/10.1016/j.jpharm.2018.06.053.

[51] W. Wanyi, L. Lu, H. Zehan, X. Xinan, Comparison of emulsifying characteristics of different macromolecule emulsifiers and their effects on the physical properties of lycopene nanoemulsions, J. $\quad$ Dispers. Sci. Technol. 41 (2020) 618-627. https://doi.org/10.1080/01932691.2019.1610421. 
[52] D. Lerche, T. Detloff, T. Sobisch, Instability Index calculation, Dispers. Lett. 4 (2013) 1-4.

[53] C. R. Harris, K. J. Millman, S. J. van der Walt, R. Gommers, P. Virtanen, D. Cournapeau, E. Wieser, J. Taylor, S. Berg, N. J. Smith, R. Kern, M. Picus, S. Hoyer, M. H. van Kerkwijk, M. Brett, A. Haldane, J. F. del Río, M. Wiebe, P. Peterson, P. Gérard-Marchant, K. Sheppard, T. Reddy, W. Weckesser, H. Abbasi, C. Gohlke, T. E. Oliphant, Array programming with NumPy, Nature. 585 (2020) 357-362. https://doi.org/10.1038/s41586020-2649-2.

[54] W. McKinney, Data structures for statistical computing in python, in: Proc. 9th Python Sci. Conf., 2010: pp. 51-56.

[55] J. D. Hunter, Matplotlib: A 2D graphics environment, Comput. Sci. Eng. 9 (2007) 90-95.

[56] V. Baric, H. K. Grossmann, W. Koch, L. Mädler, Quantitative Characterization of Mixing in Multicomponent Nanoparticle Aggregates, Part. Part. Syst. Charact. 35 (2018) 1-13. https://doi.org/10.1002/ppsc.201800177.

[57] D. Lerche, T. Sobisch, Evaluation of particle interactions by in situ visualization of separation behaviour, Colloids Surfaces A Physicochem. Eng. Asp. 440 (2014) 122-130. https://doi.org/10.1016/j.colsurfa.2012.10.015.

[58] T. Sobisch, D. Lerche, Separation behaviour of particles in biopolymer solutions in dependence on centrifugal acceleration: Investigation of slow structuring processes in formulations, Colloids Surfaces A Physicochem. Eng. Asp. 536 (2018) 74-81. https://doi.org/10.1016/j.colsurfa.2017.07.038.

[59] A. V Butenko, P. M. Nanikashvili, D. Zitoun, E. Sloutskin, Critical onset of layering in 
sedimenting suspensions of nanoparticles, Phys. Rev. Lett. 112 (2014) 188301.

[60] M. J. Uttinger, S. Boldt, S. E. Wawra, T. D. Freiwald, C. Damm, J. Walter, D. Lerche, W. Peukert, New Prospects for Particle Characterization Using Analytical Centrifugation with Sector-Shaped Centerpieces, Part. Part. Syst. Charact. (2020) 2000108.

[61] R. R. Retamal Marín, F. Babick, G. G. Lindner, M. Wiemann, M. Stintz, Effects of sample preparation on particle size distributions of different types of silica in suspensions, Nanomaterials. 8 (2018). https://doi.org/10.3390/nano8070454.

[62] G. J. Price, F.S. Paul, Ultrasonic degradation of polymer solutions: 2 . The effect of temperature, ultrasound intensity and dissolved gases on polystyrene in toluene, Polymer (Guildf). 34 (1993) 4111-4117.

[63] A. V Mohod, P. R. Gogate, Ultrasonics Sonochemistry Ultrasonic degradation of polymers : Effect of operating parameters and intensification using additives for carboxymethyl cellulose (CMC) and polyvinyl alcohol (PVA), Ultrason. - Sonochemistry. 18 (2011) 727734. https://doi.org/10.1016/j.ultsonch.2010.11.002.

[64] I. M. Mahbubul, Preparation, Characterization, Properties, and Application of Nanofluid, 1st editio, William Andrew, 2018. https://doi.org/10.1016/B978-0-12-813245-6.00003-4.

[65] V. S. Nguyen, D. Rouxel, R. Hadji, B. Vincent, Y. Fort, Ultrasonics Sonochemistry Effect of ultrasonication and dispersion stability on the cluster size of alumina nanoscale particles in aqueous solutions, Ultrason. - $\quad$ Sonochemistry. $18 \quad$ (2011) 382-388. https://doi.org/10.1016/j.ultsonch.2010.07.003.

[66] E. Y. Safronova, G. Pourcelly, A. B. Yaroslavtsev, The transformation and degradation of 
Nafion ${ }^{\circledR}$ solutions under ultrasonic treatment. The effect on transport and mechanical properties of the resultant membranes, Polym. Degrad. Stab. 178 (2020) 109229. https://doi.org/10.1016/j.polymdegradstab.2020.109229.

[67] W. Peukert, D. Segets, L. Pflug, G. Leugering, Unified design strategies for particulate products, in: Adv. Chem. Eng., Elsevier, 2015: pp. 1-81.

[68] H. Rumpf, Grundlagen und Methoden des Granulierens, Chemie Ing. Tech. 30 (1958) 144158.

[69] H. M. Santos, C. Lodeiro, J. L. Capelo-Martínez, The power of ultrasound, in: Ultrasound Chem. Anal. Appl., Wiley Online Library, 2009: pp. 1-16.

[70] M. Adamski, N. Peressin, S. Holdcroft, B. G. Pollet, Does power ultrasound affect Nafion® $\begin{array}{lllll}\text { dispersions?, } & \text { Ultrason. } & \text { Sonochem. } & 60 & (2020)\end{array}$ https://doi.org/10.1016/j.ultsonch.2019.104758.

\section{Figure Captions}

Figure 1: Schematic showing the novel characterization toolbox introduced in this work to study fuel cell inks using AC. (A) Working principle of AC. Light transmitted through the sample cell over a radial position of ri to $\mathrm{rf}$ from the rotor center is captured by CCD detectors at defined time intervals. (B) Schematic structure of a PEMFC ink composed of a carbon-supported Pt catalyst and ionomer. (C) Transmittogram illustrating the time and space resolved changes in attenuated transmission values. The initial ri and the final $\mathrm{rf}$ radial position of the sample from the rotor center and elapsed time are indicated on the $\mathrm{y}$ - and $\mathrm{x}$ - axes, respectively. A grey value is assigned to 
instantaneous transmission values to construct the heatmap. (D) Stability trajectory illustrating the $\mathrm{S}$ score derived from instantaneous transmission values over elapsed time. 12

Figure 2: TEM micrographs at different magnifications, $60 \mathrm{Kx}$ (left panels), $100 \mathrm{Kx}$ (middle panels), and $300 \mathrm{Kx}$ (right panels). (A-C) Images of the catalyst ink sample UB. (D-F) Images of the catalyst ink sample UB+S20. (G-I) TEM images of the catalyst ink sample UB+S70. Some representative areas potentially covered with agglomerates are shown in white circles. 21

Figure 3: Transmission fingerprints for catalyst ink samples MS, UB, UB+S20, and UB+S70, respectively. The first profile is colored as violet, and the last profile as yellow. The meniscus for ink sample MS is shown by a black arrow at a radial position of $110 \mathrm{~mm}$. Inks were centrifuged at $4000 \mathrm{rpm}$ at $2300 \mathrm{~g}$ for about 16 hours. 22

Figure 4: (A) Transmittogram for catalyst ink sample MS. The air-liquid interface and sedimentation front are marked by a black arrow. Three aggregates / agglomerates were clearly observed settling at different speeds resulting in complete sedimentation within 4 hours. Transmittograms for ink samples (B) UB, (C) UB+S20 and (D) UB+S70 respectively. Quantilebased mapping was used for constructing transmittograms for better visualization. The radial positions for which sedimentation rates of topmost (RT) and bottommost (RB) assemblages were calculated are indicated by white lines, while corresponding transmission values are provided as part of the color code. The distance traveled from the start of the experiment (i.e., at the meniscus)

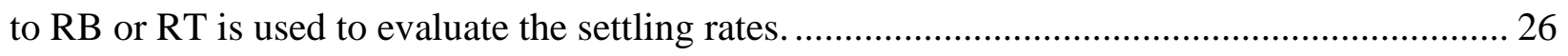
Figure 5: (A) Stability trajectory and (B) transmittogram of ink sample MS. The bulk sedimentation is achieved in about $105 \mathrm{~min}$ as indicated by a blue vertical dotted line. The continuous phase is clarified within $300 \mathrm{~min}$ as shown by the lower S score line. (C) Stability 
trajectory and (D) transmittograms of ink sample UB+S20. The BST of 805 min is indicated by a blue vertical dotted line 32

Figure 6. Stability trajectory for catalyst ink samples (A) MS (dark blue line), (B) UB (brown line), (C) $\mathrm{UB}+\mathrm{S} 20$ (magenta line) and (D) UB+S70 (dark green line), respectively. Corresponding blue dotted lines and inverted triangles show the global maxima indicating the BST. 34

Figure 7. BSTs for catalyst ink samples MS (dark blue), UB (brown), UB+S20 (magenta) and $\mathrm{UB}+\mathrm{S} 70$ (dark green) respectively. Hollow squares, hollow rectangles and hollow triangles show triplicate measurements of the corresponding ink preparation method. The filled diamonds show the means of triplicates for sedimentation times of three different inks. The mean of all measurements is shown by the horizontal colored lines along with the standard error of the means.

Figure 8. Viscosity-shear rate curves for catalyst ink sample MS (dark blue square), UB (brown circle), UB+S20 (magenta triangle) and UB+S70 (dark green diamond) respectively. Corresponding colored ribbons are made by joining the standard deviations of three measurements.

$\begin{array}{ll}\text { Abbreviations } & \text { Analytical centrifugation } \\ \text { AC } & \text { Bulk sedimentation time } \\ \text { BST } & \text { Catalyst layer } \\ \text { CL } & \text { Catalyst coated membrane } \\ \text { CCM } & \text { Catalyst coated layer } \\ \text { CCL } & \text { Dynamic light scattering }\end{array}$


DLVO Derjaguin-Landau-Verwey-Overbeek

GDL Gas diffusion layer

HOR Hydrogen oxidation reaction

IPA Isopropyl alcohol

MEA Membrane electrode assembly

ORR Oxidation reduction reaction

PEMFC Proton electrolyte membrane fuel cell

RCF Relative centrifugal force

ROI Region of interest

STEPTM Space- and Time-resolved Extinction Profiles

TEM Transmission electron microscopy 